International Journal of Modern Physics B Vol. 29, No. 32 (2015) 1599001 (26 pages) (c) World Scientific Publishing Company DOI: $10.1142 / S 0217979215990015$

\title{
AUTHOR INDEX Volume 29
}

Abadyan, M., see Farrokhabadi, A.

Abbasi, F. M., Hayat, T. \& Alsaadi, F., Hydromagnetic peristaltic transport of water-based nanofluids with slip effects through an asymmetric channel

Abd Fatah, A. Y., Mazlan, S. A., Koga, T., Zamzuri, H., Zeinali, M. \& Imaduddin, F., A review of design and modeling of magnetorheological valve

Abdallah, A. \& Benrekaa, N., Study of the free volume fraction in polylactic acid (PLA) by thermal analysis

Abdullah, A. H., see Nik Roselina, N. R.

Abolhassani, M. R., see Taghavi Mendi, R.

Abraime, B., see Ait-Tamerd, M.

Abu Hassan, H., see Zandi Goharrizi, A.

Abu-Jafar, M. S., see El-Kelma, R. Z.

Abu-Jafar, M. S., see Khalfa, M.

Abu-Jafar, M. S., see Mahmoud, N. T.

Acosta, J. G., Joya, M. R. \& Barba-Ortega, J., Neutral donor in a bilayer spherical semiconductor quantum dot

Adhikari, N. P., see Pantha, N.

Agafonov, A. I., Phonon residual resistance of pure crystals

Agop, M., Gavriluţ, A. \& Rezuş, E., Implications of Onicescu's informational energy in some fundamental physical models

Ahl Laamara, R., see Daoud, M.

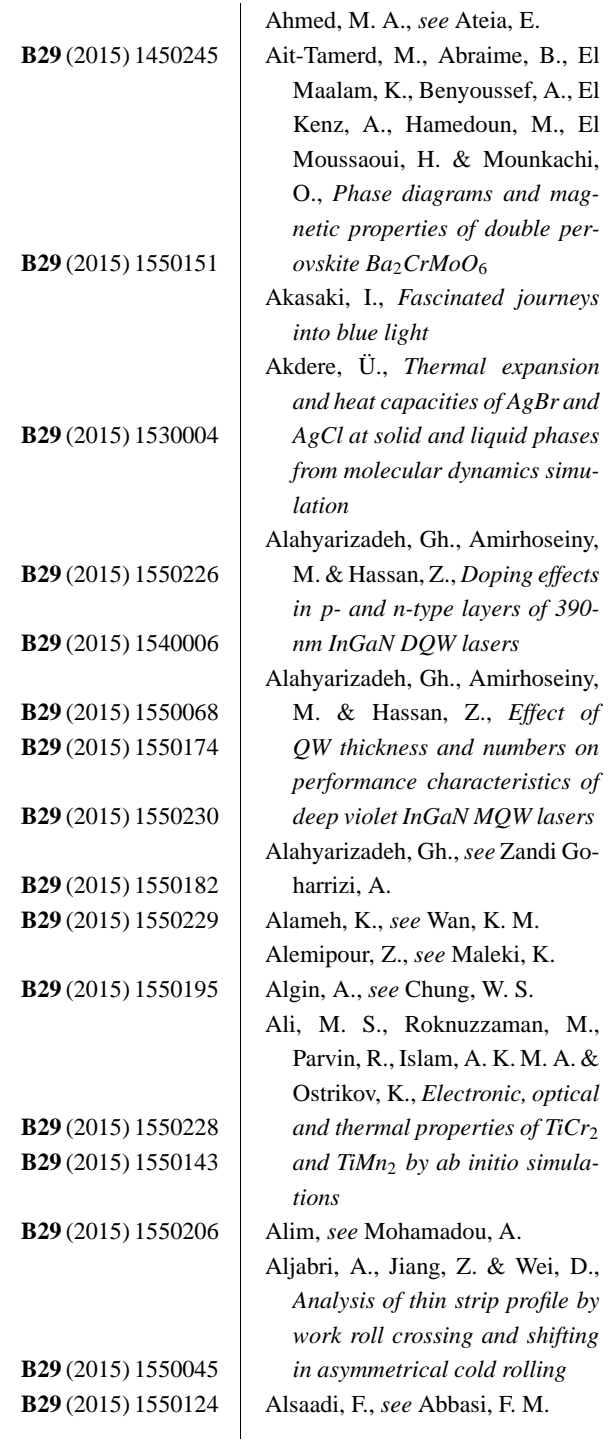

B29 (2015) 1550126

B29 (2015) 1550174

B29 (2015) 1530014

B29(2015) 1550091

B29(2015) 1550118

B29(2015) 1550081

B29 (2015) 1550230

B29 (2015) 1550238

B29 (2015) 1550224

B29 (2015) 1550177

B29 (2015) 1550223

B29 (2015) 1550049

B29 (2015) 1540032 B29(2015) 1550151 
Altındal, Ş., see Tecimer, H.

Altındal, Ş., see Yücedăg, İ.

Altındal, Ş., see Çetinkaya, H. G.

Amakai, Y., see Shimizu, M.

Amano, H., Growth of GaN on sapphire via low-temperature deposited buffer layer and realization of p-type GaN by $\mathrm{mg}$ doping followed by low-energy electron beam irradiation

Amellal, H., Meslouhi, A. \& Hassouni, Y., On the deformed cat state: Generating scheme via amplitude dispersion and the analysis of nonclassical features

Amirhoseiny, M., see Alahyarizadeh, Gh.

Amirhoseiny, M., see Alahyarizadeh, Gh.

Amrani, B., see Belarbi, W. T.

Andreev, P. A. \& Kuz'menkov, L. S., Waves of spin current in magnetized dielectrics

Anwar, M., Khan, Z. S., Mustafa, K. \& Rana, A., Effects of some rare earth and carbonate-based co-dopants on structural and electrical properties of samarium doped ceria (SDC) electrolytes for solid oxide fuel cells

Ao, Q. B., see Wang, J. Z.

Ao, Y.-Y., see Zhang, X.-K

Arabi, H., see Roohani, E.

Artunç, N., see Cetinel, A.

Askerzade, I. N., see Rakhimov, A.

Ataie, A., see Hassanirad, A.

Ataie, A., see Jahangir, S.

Ateia, E., Ahmed, M. A. \& Ghouniem, R. M., Effect of rare earth substitution on the structural and electrical properties of $\mathrm{Cu}-\mathrm{Mg}$ ferrite

Audzijonis, A., Žigas, L., Žaltauskas, R. \& Sereika, R., Origin of ferroelectric phase transition in $\mathrm{SbO}_{x} S_{1-x} I$ mixed crystals

Azizan, A., see Nik Roselina, N. R.

Baar, S., see Shimizu, M.

Babu, B. R., Prasad, M. S. R. \& Ramesh, K. V., Effect on struc-
B29 (2015) 1550076
B29 (2015) 1550075
B29 (2015) 1450237
B29 (2015) 1542014

B29 (2015) 1530015

B29 (2015) 1550232

B29(2015) 1550118

B29 (2015) 1550081

B29 (2015) 1550056

B29 (2015) 1550077

B29 (2015) 1550179

B29 (2015) 1540002

B29 (2015) 1550029

B29 (2015) 1550190

B29 (2015) 1550093

B29 (2015) 1550123

B29 (2015) 1592003

B29 (2015) 1592002

B29 (2015) 1550126

B29 (2015) 1550167

B29 (2015) 1540006 B29 (2015) 1542014 tural and magnetic properties of aluminum substituted $\mathrm{Ni}-\mathrm{Zn}$ nanoferrite system prepared via citrate-gel route

Babu, B. R., see Prasad, M. S. R.

Bae, J. J., see Bok, J. M.

Bagayoko, D., see Malozovsky, Y.

Bai, S., see Qin, J.

Bai, S., see Zhang, W.

Bai, Y. H., see Zhang, G. P.

Bai, Y., Luan, M., Li, L., He, Z. \& Li, D., The sensitization of laser-induced heat of $\mathrm{Er}^{3+}$ doped $\mathrm{Y}_{2} \mathrm{O}_{3}$ nanocrystals by codoped with $\mathrm{Mn}^{2+}$

Bai, Z., see Miao, R.

Baki, N., see Khalfa, M.

Bang, W., Teizer, W., Rathnayaka, K. K. D., Lyuksyutov, I. F. \& Naugle, D. G., Controlling superconductivity in thin film with an external array of magnetic nanostructures

Bang, W., Teizer, W., Rathnayaka, K. K. D., Lyuksyutov, I. F. \& Naugle, D. G., Using electrochemical fabrication to grow external arrays of magnetic nanostripes to manipulate superconductivity in the thin film

Bao, K., see Wang, X. P.

Bao, T. F., see Wang, J. Z.

Baoxing, L., see Zhihua, $P$.

Barba-Ortega, J., see Acosta, J. G.

Barrera, E. W., see Jaramillo, J. A. $P$

Belarbi, W. T., Rouabhia, A., Tair, F., Amrani, B. \& Sekkal, N., Low symmetry phases of ( $\mathrm{Al}$, $G a)$ Sb under low pressure

Beldi, L., see El-Kelma, R. Z.

Belhadji, Y., Bouazza, B., Moulahcene, F. \& Massoum, N., Ensemble Monte Carlo analysis of subpicosecond transient electron transport in cubic and hexagonal silicon carbide for high power SiC-MESFET devices

Belkhiat, S., see Madani, L.

Belobo Belobo, D., see Mboumba, M. D.

Belogorlov, A. A., see Borman, V. D.

Ben-Bolie, G. H., see Mboumba,
B29 (2015) 1550032

B29 (2015) 1550067

B29 (2015) 1542005

B29 (2015) 1542006

B29 (2015) 1540027

B29 (2015) 1540026

B29 (2015) 1550115

B29 (2015) 1550158 B29 (2015) 1550064 B29 (2015) 1550229

B29 (2015) 1542035

B29 (2015) 1542036 B29 (2015) 1550106 B29 (2015) 1540002 B29 (2015) 1550105 B29 (2015) 1550228

B29 (2015) 1550217

B29 (2015) 1550056

B29 (2015) 1550182

B29 (2015) 1550107 B29 (2015) 1550186

B29 (2015) 1550008

B29 (2015) 1550097 
Author Index

M. D.

Ben-Bolie, G. H., see Mboumba, M. D.

Benrekaa, N., see Abdallah, A.

Benyoussef, A., see Ait-Tamerd, M.

Berrag, A., see Madani, L.

Bharadwaj, S., see Swamy, D. R. S. G.

Bhattacharjee, J. K., see Pal, S.

Bhattacharyya, D., see Tran, L. Q. N.

Bhattacharyya, D., see Wu, H.

Bhattacherjee, A. B. \& De, S., Frequency measurement using Rayleigh scattering from a $B E C$

Bian, B., Chu, B., Zhu, Z. \& Zheng, Y., Effect of inserted $\mathrm{Cu}$ layer on $\mathrm{CoFe} / \mathrm{Cu} / \mathrm{MgO} / \mathrm{CoFe}$ magnetic tunnel junction

Bin Omran, S., see Khalfa, M.

Bittner, E. R., see Zaster, S.

Bjurenstedt, A., Seifeddine, S. \& Jarfors, A. E. W., On the complexity of the relationship between microstructure and tensile properties in cast aluminum

Bodrova, A., see Stadnichuk, V.

Boekema, C., Owens, F., Love, A., Li, Z., Sakkaris, P. \& Dawson, W. K., A magnetic origin of cuprate superconductivity? A MaxEnt- $\mu S R$ view

Bok, J. M., Bae, J. J., Hong, S. H., Zhou, X. J. \& Choi, HY., Low energy impurity kink in the normal and anomalous self-energies in Bi-cuprate superconductors

Bonacum, J., see Zhang, G. P.

Bonalde, I., see Macias-M, A.

Bondarenko, S. \& Koverya, V., Superconductivity in the Institute for Low Temperature Physics and Engineering of the $\mathrm{Na}$ tional Academy of Sciences of Ukraine

Boochani, A., see Jafari, A.

Boochani, A., see Taghavi Mendi, $\mathrm{R}$.

Bordbar, G. H. \& Sabet, M. T. M., A variational calculation of magnetized correlated fermion
B29 (2015) 1550202

B29 (2015) 1550008 B29 (2015) 1550226

B29 (2015) 1550174 B29 (2015) 1550186

B29 (2015) 1550218 B29 (2015) 1550216

B29 (2015) 1540018

B29 (2015) 1540019

B29 (2015) 1550051

B29 (2015) 1550171

B29 (2015) 1550229

B29 (2015) 1550157

B29 (2015) 1540011

B29 (2015) 1550208

B29 (2015) 1542026

B29 (2015) 1542005

B29 (2015) 1550115 B29 (2015) 1550150

B29 (2015) 1542013 B29 (2015) 1550133

B29 (2015) 1550068 system using a spin-dependent correlation: Application to liquid ${ }^{3} \mathrm{He}$

Borkar, H., Seifeddine, S. \& Jarfors, A. E. W., Microstructure analysis of $\mathrm{Al}-\mathrm{Si}-\mathrm{Cu}$ alloys prepared by gradient solidification technique

Borman, V. D., Belogorlov, A. A., Grekhov, A. M. \& Tronin, V. N., States of a dispersed nonwetting liquid in a disordered nanoporous medium

Bouazza, B., see Belhadji, Y.

Bouchriha, H., see Ridene, R.

Bouchriha, H., see Saidi, H.

Bougherara, K., see Khalfa, M.

Bouhafs, B., see El-Kelma, R. Z.

Boulechfar, R., Meradji, H., Chouahda, Z., Ghemid, S., Drablia, S. \& Khenata, R., FP-LAPW investigation of the structural, electronic and thermodynamic properties of $\mathrm{Al}_{3} \mathrm{Ta}$ compound

Bozovic, I., see Gasparov, V. A.

Brilliantov, N., see Stadnichuk, V.

Buksman, E., de Oliveira, A. L. F. \& de Lacalle, J. G. L., Search via quantum walks with intermediate measurements

Bulaeva, E. A., see Pankratov, E. L.

Cabra, D., see Rodríguez-Ponte, P.

Cai, J., see Tan, X.-H.

Cai, L., see Cui, H.

Cai, L.-C., see Guo, Z.-C.

Cai, X., Pan, H. \& Wang, Z. S., Geometric phase of two-qubit system in dephasing environment

Cai, X.-J., see Wang, C.-D.

Can, D., see Miao, R.

Cao, G. \& Han, Y., Does the weather affect the Chinese stock markets? Evidence from the analysis of DCCA crosscorrelation coefficient

Cao, G., Zhao, Y. \& Han, Y., Asymmetric statistical features of the Chinese domestic and international gold price fluctuation

Cao, G., see Chen, Q.
B29 (2015) 1550046

B29(2015) 1540015

B29 (2015) 1550097

B29 (2015) 1550107

B29 (2015) 1550211

B29 (2015) 1550054

B29 (2015) 1550229

B29 (2015) 1550182

B29 (2015) 1450244

B29 (2015) 1542012

B29 (2015) 1550208

B29 (2015) 1550127

B29 (2015) 1550023

B29 (2015) 1550102

B29 (2015) 1550135

B29 (2015) 1550203

B29 (2015) 1550096

B29 (2015) 1550236

B29 (2015) 1540031

B29 (2015) 1550064

B29 (2015) 1450236

B29 (2015) 1550113

B29 (2015) 1542050 
Cao, L. P., Pan, L. Q., Li, W. M., Wang, X. C., Liu, Q. Q., Long, Y. W., Gu, C. Z. \& Jin, C. Q., High pressure growth and characterization of $\mathrm{SrCrO}_{3}$ single crystal

Cao, P., see Hayat, M. D.

Cao, P., see Huang, C.

Cao, P., see Ouyang, X.

Cao, P., see Xu, Z.

Cao, R., see Gao, F.

Cao, X., Che, Y. \& Yao, J., Resonant conversion based on GaAs-metal metamaterials within terahertz range

Çataltepe, Ö. A., see Özdemir, Z. G.

Ceng, S., see Liu, Y.

Çetinkaya, H. G., Yıldız, D. E. \& Ş. Altındal On the negative capacitance behavior in the forward bias of $\mathrm{Au} / \mathrm{n}-4 \mathrm{H}-$ $\mathrm{SiC}(\mathrm{MS})$ and comparison between $\mathrm{MS}$ and $\mathrm{Au} / \mathrm{TiO}_{2} / n-4 \mathrm{H}_{-}$ $\mathrm{SiC}$ (MIS) type diodes both in dark and under $200 \mathrm{~W}$ illumination intensity

Cetinel, A., Artunç, N., Sahin, G. \& Tarhan, E., Influence of applied current density on the nanostructural and light emitting properties of n-type porous silicon

Chainok, P., Khuntak, T., Sujinnapram, S., Tiyasri, S., Wongphakdee, W., Kruaehong, T., Nilkamjon, T., Ratreng, S. \& Udomsamuthirun, P., Some properties of $\mathrm{YBa}_{m} \mathrm{Cu}_{1+m} \mathrm{O}_{y}$ $(m=2,3,4,5)$ superconductors

Chan, K. S., see Lee, C. M.

Chand, S., Singh, R. P., Govindan, A. \& Singh, S. K., Mechanical, spin polarized electronic and magnetic properties of $\operatorname{TmX}$ $(X=C u, A g)$ : First principle study

Chang, H. W., see Lee, Y. I.

Chang, J., see Wu, X.-L.

Chang, W. C., see Lee, Y. I.

Chanpoom, T., Effect of pseudogap on the specific heat of superconductor

Chaudhury, R., see Sarkar, S.
B29 (2015) 1542025

B29 (2015) 1540005

B29 (2015) 1540017

B29 (2015) 1540003

B29 (2015) 1540014

B29 (2015) 1550192

B29 (2015) 1550145

B29 (2015) 1550205

B29(2015) 1540029

B29(2015) 1450237

B29 (2015) 1550093

B29 (2015) 1550060 B29 (2015) 1550037

B29 (2015) 1550007 B29 (2015) 1540007 B29 (2015) 1550103 B29 (2015) 1540007

B29 (2015) 1550180 B29 (2015) 1550209
Chaudhury, R., see Sarkar, S.

B29 (2015) 1592004

Che, T., Gou, Y. F., Deng, Z. G., Zheng, J., Zheng, B. T. \& Chen, P., A method to enhance the curve negotiation performance of HTS Maglev

B29 (2015) 1542037

Che, Y., Yang, T., Li, R., Li, H., Han, C., Wang, J. \& Wei, $\mathrm{X}$., Desynchronization in an ensemble of globally coupled chaotic bursting neuronal oscillators by dynamic delayed feedback control

Che, Y., see Cao, X.

Chemam, R., see Graine, R.

Chen, B. S., see Yin, J.

Chen, C., see Zha, D.

Chen, H., Wu, R., Huang, G. \& Deng, H., Dimer-monomer model on the towers of Hanoi graphs

B29 (2015) 1550173

B29 (2015) 1542049

B29 (2015) 1540029

B29 (2015) 1550162

B29 (2015) 1540025

B29 (2015) 1540033

B29 (2015) 1550220

B29 (2015) 1550139

Chen, J., see You, F.

Chen, L. A., Zhu, X. F. \& Chen, L. F., First principles investigation of the diffusion of interstitial $\mathrm{Cu}, \mathrm{Ag}$ and $\mathrm{Au}$ in $\mathrm{ZnTe}$

Chen, L. F., see Chen, L. A.

Chen, L., see Chen, Y.

Chen, L., see Wu, Z.

Chen, M., see Wang, C.-D.

Chen, P., see Che, T.

Chen, Q. F., see Sun, X. N.

Chen, Q., Sun, X., Liu, A., Zhang, Q., Cao, G. \& Zhou, X., Photovoltaic performance of dyesensitized solar cells using $\mathrm{TiO}_{2}$ nanotubes aggregates produced by hydrothermal synthesis

Chen, R. H., see Li, W. X.

Chen, S., see Ren, X.

Chen, S., see Sheng, C.-H.

Chen, T. N., see Wang, X. P.

Chen, T., see Li, Y.

Chen, T., see Xiong, J.

Chen, W.-X., Xie, Y.-X. \& Xi, X.-Q., Measurement-induced nonlocality in the two-qubit Heisenberg XY model

Chen, X., see Guan, Q.
B29 (2015) 1550130 B29 (2015) 1550130 B29 (2015) 1550134 B29 (2015) 1550213 B29 (2015) 1540031 B29 (2015) 1542037 B29 (2015) 1542044

B29 (2015) 1542050 B29 (2015) 1542032 B29 (2015) 1550220 B29 (2015) 1550066 B29 (2015) 1550106 B29 (2015) 1550002 B29 (2015) 1550191

B29 (2015) 1550098 B29 (2015) 1550128 
Author Index

Chen, X., see Leng, M.

Chen, X., see Li, L.

Chen, X., see Zhang, W.

Chen, X.-R., see Yu, J.-X.

Chen, Y. B., see Hou, Q. R.

Chen, Y. J., see Lee, Y. I.

Chen, Y., Gu, X., Jiang, D., Xie,

L. \& Chen, L., Counterfactual quantum cryptography network with untrusted relay

Chen, Y., Ma, R., Wang, K., Gao, F., Hu, X. \& Song, H., Thermoelectric properties of holedoped $\mathrm{Yb}_{1-x} \mathrm{~Pb}_{x} \mathrm{BaCO}_{4} \mathrm{O}_{7+\delta}$ ceramics

Chen, Y., Wang, Z. \& Qiu, Y., Aerosol synthesis and application of folded graphene-based materials

Chen, Y., see Yang, T

Chen, Y., see Yin, S.

Cheng, C. H., see Jiang, J.

Cheng, J., see Leng, M.

Cheng, L. F., see Wu, H.

Cheng, L., Xue, X., Tang, B., Kou, H. \& Li, J., Effect of hot-forging on beta phase transformation of a high niobium containing titanium aluminide alloy

Cheng, Y. K., see Ding, C. C.

Cheng, Y. K., see Zhang, L. J.

Cheng, Y., see Yu, J.-X.

Chi, Z., Guo, X., Wang, Z. \& Zheng, Q., Topological phase transitions of three-dimensional topological insulator without energy gap closing

Chiker, F., see Khalfa, M.

Choi, H-Y., see Bok, J. M.

Choi, J. R., Wave functions with discrete and with continuous spectrum for quantum damped harmonic oscillator perturbed by a singularity

Chouahda, Z., see Boulechfar, R.

Chu, B., see Bian, B.

Chu, R., see Ma, J.

Chu, T., see Feng, L.-Q.

Chung, S. G., see Wang, L.

Chung, W. S. \& Algin, A., q-Deformed Tamm-Dancoff oscillators: Representation, fermionic extension and physical application

Chuong, T. V., see Tuan, D. A.
B29 $(2015) 1550078$
B29 $(2015) 1550108$
B29 $(2015) 1540026$
B29 $(2015) 1450247$
B29 $(2015) 1550189$
B29 $(2015) 1540007$

B29 (2015) 1550134

B29 (2015) 1550082

B29 (2015) 1530003

B29 (2015) 1540033

B29 (2015) 1550225

B29 (2015) 1542046

B29 (2015) 1550078

B29 (2015) 1540019

B29 (2015) 1540009

B29 (2015) 1542015

B29 (2015) 1542017

B29 (2015) 1450247

B29 (2015) 1550199

B29 (2015) 1550229

B29 (2015) 1542005

B29 (2015) 1592001

B29 (2015) 1450244

B29 (2015) 1550171

B29 (2015) 1450239

B29 (2015) 1550053

B29 (2015) 1550042

B29 (2015) 1550177

B29 (2015) 1550231
Claro, F., Fuchs, R., Robles, P. \& Rojas, R., The effect of dissipation on the torque and force experienced by nanoparticles in an AC field

Cottam, M. G., see Komorowski, P. G.

Cui, H., Cai, L., Wang, S., Liu, X. \& Yang, X., Accurate reliability analysis method for quantumdot cellular automata circuits

Dadsetani, M. \& Omidi, A. R., Optical filtering properties of $\mathrm{TiO}_{2} / \mathrm{Al}_{2} \mathrm{O}_{3}$ heterostructures from first principles

Dai, H.-M., see Lu, J.-D.

Danieli, C., Rayanov, K., Pavlov, B., Martin, G. \& Flach, S., Approximating metal-insulator transitions

Daoud, M., Ahl Laamara, R. \& Seddik, S., A recursive approach for geometric quantifiers of quantum correlations in multiqubit Schrödinger cat states

Dawson, W. K., see Boekema, C. de Lacalle, J. G. L., see Buksman, E.

de Oliveira, A. L. F., see Buksman, E.

De, S., see Bhattacherjee, A. B.

Dedkov, G. \& Kyasov, A., Thermal radiation, radiation force and dynamics of a polarizable particle in vacuum

Deng, H., see Chen, H.

Deng, K., see He, H.-L.

Deng, Z. G., see Che, T.

Deng, Z. G., see Sun, R. X.

Deng, Z., see Han, W.

Deng, Z., see Xing, L. Y.

Derzhko, O., Richter, J. \& Maksymenko, M., Strongly correlated flat-band systems: The route from Heisenberg spins to Hubbard electrons

Dhillon, M., see Fang, F.

Dialameh, M., see Safarpour, Gh. Ding, B. F., see Wan, K. M.

Ding, C. C., Wu, S. Y., Cheng, Y. K. \& Zhang, L. J., Investigations of the defect structure for $\mathrm{Cu}^{2+}$ in $\mathrm{LaSrGa}_{0.995} \mathrm{Cu}_{0.005} \mathrm{O}_{4}$ ceramics
B29 (2015) 1450240

B29(2015) 1550074

B29 (2015) 1550203

B29 (2015) 1550047 B29 (2015) 1550165

B29 (2015) 1550036

B29 (2015) 1550124 B29 (2015) 1542026

B29(2015) 1550127

B29 (2015) 1550127

B29 (2015) 1550051

B29 (2015) 1550237 B29 (2015) 1550173 B29 (2015) 1550193 B29 (2015) 1542037 B29 (2015) 1542043 B29 (2015) 1550019 B29 (2015) 1542023

B29 (2015) 1530007 B29 (2015) 1540013 B29 (2015) 1550085 B29 (2015) 1550238

B29 (2015) 1542015 
Ding, C. C., see Zhang, L. J.

Ding, M., Han, J., Qiu, W., Zhang, W. \& Gao, W., Recovery of silver metal from low concentrated wastewater by photocatalysis

Dong, F.-Z., see Shu, H.-S.

Dong, H.-N., Liu, X.-S. \& Zhou, H.-F., Investigations of the EPR $g$ factors for tetragonal copper site in $\mathrm{Sr}_{2} \mathrm{Ca}_{2} \mathrm{Cu}_{3} \mathrm{O}_{x}$

Dong, H.-N., Zhou, H.-F. \& Yang, G.-R., Theoretical studies of the crystal-field energy levels and EPR $g$ factors for $\mathrm{CeFeAs} \mathrm{O}_{1-x} F_{x}$

Dong, H.-N., see He, J.-J.

Dong, H.-N., see Wang, J.

Dong, H.-N., see Xu, Y.-Q.

Dong, X.-G., see Zhang, H.-G.

Dou, S. X., see Li, W. X.

Drablia, S., see Boulechfar, R.

Dridi, Z., see Hamri, A.

Duan, G., see Wu, Y.

Duan, Y., see Shao, C.

Dubuis, G., see Gasparov, V. A

Ekogo, T. B., see Mboumba, M. D.

Ekogo, T. B., see Mboumba, M. D.

Ekuma, C., see Malozovsky, Y.

El Ghazi, H. \& Peter, A. J., Impurity-related nonlinear optical absorption under combined effects of intense laser field and band-edge nonparabolicity on InGaN $Q W$

El Ghazi, H., Intense laser field and conduction band-edge nonparabolicity effects on hydrogenic impurity states of InGaN $Q W$

El Haj Hassan, F., see El-Kelma, R. Z.

El Kenz, A., see Ait-Tamerd, M.

El Maalam, K., see Ait-Tamerd, M.

El Moussaoui, H., see Ait-Tamerd, M.

El-Kelma, R. Z., Beldi, L., El Haj Hassan, F., Murtaza, G., Khenata, R., Abu-Jafar, M. S., Omran, S. B. \& Bouhafs, B., Magnetic ordering and electronic structure of the ternary iron ar-
B29 (2015) 1542017

B29 (2015) 1540028

B29(2015) 1550176

B29 (2015) 1542011

B29 (2015) 1542022

B29 (2015) 1542019

B29 (2015) 1542021

B29 (2015) 1542016

B29 (2015) 1550006

B29 (2015) 1542032

B29 (2015) 1450244

B29 (2015) 1550057

B29 (2015) 1540001

B29 (2015) 1550131

B29 (2015) 1542012

B29 (2015) 1550202

B29 (2015) 1550008 B29 (2015) 1542006

B29 (2015) 1550110

B29(2015) 1450246

B29 (2015) 1550182 B29 (2015) 1550174

B29 (2015) 1550174

B29 (2015) 1550174 senide $\mathrm{BaFe}_{2} \mathrm{as}_{2}$

Elahi, S. M., see Taghavi Mendi, $\mathrm{R}$.

Ersöz, G., see Yücedağ, İ.

Ertaş, M., see Kantar, E.

Escobar, J., see Stashans, A.

Fan, C.-J., see Lyu, G.-D.

Fan, H., see You, F.

Fan, H.-Y., see Xu, X.-L.

Fan, J., see Xie, W.

Fan, S. W., see Wu, H.

Fang, F., Kennedy, J., Dhillon, M. \& Flint, S., Antibacterial effect of silver nanofilm modified stainless steel surface

Fang, Y.-T., He, H.-Q. \& Lin, Z.L., Nonreciprocal perfect $a b$ sorber based on an ultracompact nonsymmetry cavity structure

Fang, Y.-T., see Hu, J.-X.

Farhadi, G., see Fathi, M. B.

Farjam, M., see Mirzadeh, M.

Farooq, K., Khan, M. A., Wang, L. C. \& Yi, X. X., Dynamics and transmissivity of optomechanical system in squeezed environment

Farrokhabadi, A., Mokhtari, J., Rach, R. \& Abadyan, M., Modeling the influence of the Casimir force on the pull-in instability of nanowire-fabricated nanotweezers

Farzin, Y. A., see Mirzaee, O.

Fathi, M. B., Kanjouri, F. \& Farhadi, G., Electronic structure and elastic properties of single crystal of shape memory alloys $\mathrm{TiNi}_{(1-x)} \mathrm{Cu}_{x}$ : An ab initio study

Fei, S., see Zhang, X.-K.

Feng, L.-Q. \& Chu, T., Cluster assisted generation of the isolated attosecond sources

Feng, L.-Q. \& Liu, H., Water window attosecond sources generation by using the multi-cycle time-delay two circularly polarized pulses

Feng, P. \& Xie, J., Light-induced coherent magnon excitation in monolayer magnetic nanodots

Feng, S. M., see Li, W. M.

Feng, S. M., see Xing, L. Y.
B29 (2015) 1550182

B29 (2015) 1550068

B29 (2015) 1550075

B29 (2015) 1550141

B29 (2015) 1550094

B29 (2015) 1550095

B29 (2015) 1550139

B29 (2015) 1550169

B29 (2015) 1542042

B29 (2015) 1540019

B29 (2015) 1540013

B29 (2015) 1550001

B29 (2015) 1550159

B29 (2015) 1550152

B29 (2015) 1550198

B29 (2015) 1550201

B29 (2015) 1450245

B29 (2015) 1550090

B29 (2015) 1550152 B29 (2015) 1550029

B29 (2015) 1550053

B29 (2015) 1550170

B29 (2015) 1550055

B29 (2015) 1542024

B29 (2015) 1542023 
Feng, S., Lan, Y., Zhao, H., Kuang, L., Qin, L. \& Ma, X., Kineticenergy-driven superconductivity in cuprate superconductors

Feng, S., see Kuang, L.

Firoozi, A. \& Mohammadi, A., Design of plasmonic backcontact nanogratings for broadband and polarizationinsensitive absorption enhancement in thin-film solar cell

Fisher, M. E., Renormalization group theory, the epsilon expansion and Ken Wilson as I knew him

Fisher, M. E., Statistical physics in the oeuvre of Chen Ning Yang

Flach, S., see Danieli, C.

Flint, S., see Fang, F.

Franklin, L., see Malozovsky, Y. Friedman, B. A. \& Levine, G. C., Entanglement entropy of fractional quantum Hall systems with short range disorder

Fu, G., see Shen, L.

Fuchs, R., see Claro, F.

Fuentes, C., see Tran, L. Q. N.

Fujihara, J., see Nishimoto, N.

Gabbitas, B., see Yang, F.

Gamra, D., see Ridene, R.

Gao, F., He, Q., Cao, R., Wu, F., Hu, X. \& Song, H., Enhanced thermoelectric properties of the hole-doped $\mathrm{Bi}_{2-x} \mathrm{~K}_{x} \mathrm{Sr}_{2} \mathrm{Co}_{2} \mathrm{O}_{y}$ ceramics

Gao, F., see Chen, Y.

Gao, G. Y., see Hussain, M. K.

Gao, L., Wang, F., Jiang, L., Qu, L. \& Lu, Y., Controlling the excitation process of free electrons by a femtosecond elliptically polarized laser

Gao, M., see Yang, Z.

Gao, N., Wu, J. H. \& Yu, L., Large band gaps in two-dimensional phononic crystals with selfsimilarity structure

Gao, T. F., Wang, L. F., Huang, R. Z. \& Zheng, Z. G., Transport performance of feedbackcoupled Brownian ratchets with closed-loop control strategy

Gao, T., see Guan, Q.

Gao, T., see Wei, X.-H.

Gao, W., see Ding, M.
B29 (2015) 1530009 B29 (2015) 1542001

B29 (2015) 1550111

B29 (2015) 1530006

B29 (2015) 1530013

B29 (2015) 1550036

B29 (2015) 1540013

B29 (2015) 1542006

B29 (2015) 1550065

B29 (2015) 1550027

B29 (2015) 1450240

B29 (2015) 1540018

B29 (2015) 1550215

B29 (2015) 1540004

B29 (2015) 1550211

B29 (2015) 1550192

B29 (2015) 1550082

B29 (2015) 1550175

B29 (2015) 1550033 B29 (2015) 1550207

B29 (2015) 1550017

B29 (2015) 1550069 B29 (2015) 1550128 B29 (2015) 1550014 B29 (2015) 1540028
Gao, W., see Ouyang, X.

Gao, W., see Tay, S. L.

Gao, W., see Wang, S.-J.

Gao, W., see Wang, Y.

Gao, W., see Wei, X.

Garg, A., Vijayaraghavan, V., Wong, C. H., Tai, K., Singru, P. M., Mahapatra, S. S. \& Sangwan, K. S., Investigation of mechanical strength of $2 D$ nanoscale structures using a molecular dynamics based computational intelligence approach

Gasmi, F. Z., see Graine, R.

Gasparov, V. A., He, X., Dubuis, G., Pavuna, D., Kushch, N. D., Yagubskii, E. B., Schlueter, J. A. \& Bozovic, I., Magnetic field, frequency and temperature dependence of complex conductance of ultrathin $\mathrm{La}_{1.65} \mathrm{Sr}_{0.45} \mathrm{CuO}_{4} / \mathrm{La}_{2} \mathrm{CuO}_{4}$ films and the organic superconductors $\quad \kappa-(B E D T$ $\mathrm{TTF})_{2} \mathrm{Cu}\left[\mathrm{N}(\mathrm{CN})_{2}\right] \mathrm{Br}$

Gavriluţ, A., see Agop, M.

Gedam, V., see Pansari, A.

George, T. F., see Zhang, G. P.

Georgiev, D., Monte Carlo simulation of quantum Zeno effect in the brain

Ghasemi, A., see Mirzaee, O.

Ghemid, S., see Boulechfar, R.

Ghoranneviss, M., see Jafari, A.

Gökçen, M. \& Yıldırım, M., The effects of $\mathrm{Bi}_{4} \mathrm{Ti}_{3} \mathrm{O}_{12}$ interfacial ferroelectric layer on the dielectric properties of $\mathrm{Au} / \mathrm{n}-\mathrm{Si}$ structures

Ghouniem, R. M., see Ateia, E.

Gonczarek, A., see Gonczarek, R.

Gonczarek, R., Krzyzosiak, M., Gonczarek, A. \& Jacak, L., New classes of integrals inherent in the mathematical structure of extended equations describing superconducting systems

Gong, Y. M., see Jiang, J.

Gong, Y. M., see Jiang, J.

Gorev, V. N. \& Sokolovsky, A.

I., Plasma kinetic coefficients with account for relaxation processes

Gou, G., see Liu, Y.
B29 (2015) 1540003

B29 (2015) 1540010

B29 (2015) 1540022

B29 (2015) 1540008

B29 (2015) 1540021

B29 (2015) 1450242

B29 (2015) 1550028

B29 (2015) 1542012

B29 (2015) 1550045

B29 (2015) 1550149

B29 (2015) 1550115

B29 (2015) 1550039

B29 (2015) 1550090

B29 (2015) 1450244

B29 (2015) 1550133

B29(2015) 1550120

B29 (2015) 1550126

B29 (2015) 1550117

B29 (2015) 1550117

B29 (2015) 1542046

B29 (2015) 1542047

B29 (2015) 1550233

B29(2015) 1540029 
Gou, Y. F., see Che, T.

Gou, Y. F., see Sun, R. X.

Goudarzi, H., see Khezerlou, M.

Govindan, A., see Chand, S.

Graine, R., Chemam, R., Gasmi, F. Z., Nouri, R., Meradji, H. \& Khenata, R., First principles calculations of structural, electronic and optical properties of InN compound

Grandi, N., see Rodríguez-Ponte, P.

Grekhov, A. M., see Borman, V. D.

Grigorenko, I. \& Ya. Kezerashvili, R., Superfluidity of electronhole pairs between two critical temperatures

Gu, B. F., see Hou, Q. R.

Gu, C. Z., see Cao, L. P.

Gu, H., see Jia, Y.

Gu, J. J., see Han, W.

Gu, W., see Ma, X.

Gu, X., see Chen, Y.

Guan, D., Wu, J. H., Jing, L. \& Lu, K., Lattice Boltzmann simulation of acoustic resistance in microchannels

Guan, J., see Li, L.

Guan, Q., Gao, T., Shen, Y., Ma, S., Lu, T., Chen, X., Xiao, C. \& Long, X., First-principles study of electronic, dynamical and thermodynamic properties of $\gamma$ $\mathrm{Li}_{4} \mathrm{SiO}_{4}$

Güler, E. \& Güler, M., Theoretical prediction of the structural, elastic, mechanical and phonon properties of bismuth telluride under pressure

Güler, M., see Güler, E.

Gümüş, A., see Yücedağ, İ.

Guo, Z.-C., Luo, F., Zhang, X.-L., Liu, C.-A. \& Cai, L.-C., Phase transition and thermodynamic properties of beryllium telluride under high pressure

Guo, C. Q., Yan, Y., Li, L., Han, J. X., Wei, K., Lin, F. \& Zhang, H., Stable local structure in LSMCO system and its relationship with pseudogap

Guo, C. Q., see Li, L.

Guo, J., see Liu, G.

Guo, W. L., Zhang, L. L., Luo, M.
B29 (2015) 1542037

B29 (2015) 1542043

B29 (2015) 1550018

B29 (2015) 1550007

B29 (2015) 1550028

B29 (2015) 1550102

B29 (2015) 1550097

B29 (2015) 1550188

B29(2015) 1550189

B29 (2015) 1542025

B29 (2015) 1550142

B29 (2015) 1550019

B29 (2015) 1450248

B29(2015) 1550134

B29 (2015) 1550104 B29 (2015) 1550072

B29(2015) 1550128

B29 (2015) 1550222

B29 (2015) 1550222

B29 (2015) 1550075

B29 (2015) 1550096

B29 (2015) 1542028 B29 (2015) 1542004 B29 (2015) 1550004
\& Zhang, X. R., Structures and stabilities of $\left(O s_{n} N\right)^{0, \pm}(n=7-$ 11) clusters

B29 (2015) 1550163

Guo, X., Wang, Z., Zheng, Q. \& Peng, J., Topological phases of a three-dimensional topological insulator with structure inversion asymmetry

Guo, X., see Chi, Z.

Guo, X., see Zhou, J.

Hallouche, A., see Hamri, A.

Hamedoun, M., see Ait-Tamerd, M.

Hamri, A., Dridi, Z., Hamri, B. \& Hallouche, A., $R u_{2} T i_{1-x} F e_{x} G e$ : Novel candidate for the spintronic applications

Hamri, B., see Hamri, A.

Han, C., see Che, Y.

Han, J. X., see Guo, C. Q.

Han, J. X., see Li, L.

Han, J., see Ding, M.

Han, L., see Zhang, Y.

Han, S., see Jiang, F.

Han, S., see Xie, W.

Han, S., see Yuan, H.

Han, W., Wang, X. C., Gu, J. J., Liu, Q. Q., Deng, Z. \& Jin, C. Q., Doping effect on the physical properties of $\mathrm{Li}_{x} \mathrm{Fe}_{2-x} \mathrm{As}$

Han, W.-P., see Sheng, C.-H.

Han, Y., Yang, X., Zeng, W. \& Lu, W., Quantitative analysis of the effect of $\alpha$-phase on mechanical property of Ti-6Al-4V alloy during hot working process

Han, Y., see Cao, G.

Han, Y., see Cao, G.

Hantehzadeh, M. R., see Jafari, A.

Hao, S. J., see Li, L.

Harmel, M., see Khalfa, M.

Hassan, Z., see Alahyarizadeh, Gh.

Hassan, Z., see Alahyarizadeh, Gh.

Hassan, Z., see Zandi Goharrizi, A.

Hassanirad, A., Ataie, A. \& Nikkhah-Moshaee, R., Synthesis of nano-crystalline barium hexaferrite in the presence of salt catalysts

Hassouni, Y., see Amellal, H.

Hatta, G., see Kurosawa, T.

Hayat, M. D., Wen, G. \& Cao, P.,
B29 (2015) 1550034

B29 (2015) 1550199

B29 (2015) 1550038

B29 (2015) 1550057

B29 (2015) 1550174

B29 (2015) 1550057

B29 (2015) 1550057

B29 (2015) 1450235

B29 (2015) 1542028

B29 (2015) 1542004

B29 (2015) 1540028

B29 (2015) 1550136

B29 (2015) 1542027

B29 (2015) 1542042

B29 (2015) 1550019 B29 (2015) 1550066

B29 (2015) 1540012

B29 (2015) 1550113

B29 (2015) 1450236

B29 (2015) 1550133

B29 (2015) 1542004

B29 (2015) 1550229

B29(2015) 1550118

B29 (2015) 1550081

B29 (2015) 1550230

B29 (2015) 1592003

B29 (2015) 1550232

B29 (2015) 1542009
B29 (2015) 1542010 
An easy-to-decompose binder for Ti metal injection molding: Preparation and characterization of feedstock

Hayat, T., see Abbasi, F. M.

He, H.-L., Ou-Yang, S.-L., He, Z., Deng, K. \& Zhao, H., Flexibly guiding of acoustic waves by one-dimensional sonic crystal with omnidirectional bandgap

He, H.-Q., see Fang, Y.-T.

He, J., see Liu, C.-C.

He, J., see Liu, G.

He, J., see Liu, G.

He, J.-J., Wu, S.-Y., Zhang, Z.H. \& Dong, H.-N., Studies on the $g$ factors of tetragonal $\mathrm{Cu}^{2+}$ sites in $\mathrm{NdBa}_{2} \mathrm{Cu}_{3} \mathrm{O}_{x}$ and $\mathrm{Nd}_{0.05} \mathrm{Y}_{0.95} \mathrm{Ba}_{2} \mathrm{Cu}_{3} \mathrm{O}_{6}$

He, Q., see Gao, F.

He, S., see Li, P.

He, T., Wang, Y., Sun, W. \& Zhao, $\mathrm{X}$., Effects of high magnetic field annealing on the initial recrystallized texture in pure copper

He, X., see Gasparov, V. A.

He, Z., see Bai, Y.

He, Z., see He, H.-L.

Heermann, D. W., see Máté, G.

Hikita, H. \& Morigaki, K., Diffusion coefficient using pausing time of hydrogen in a-Si:H with exponential energy distribution

Hikita, H. \& Morigaki, K., Pausing-time distribution of hydrogen diffusion in Gaussian distribution energy

Hodgson, M. A., see Xu, Z.

Hong, L. V., see Tuan, D. A.

Hong, S. H., see Bok, J. M.

Honma, T. \& Hor, P.-H., On the quantitative determination of hole-concentration in hightemperature cuprate superconductors

Hontinfinde, F., see Karimou, M. Hor, P.-H., see Honma, T.

Hou, M., Xia, Y.-Q. \& Hou, X.-W., Dynamical correlations of mutual information and tripartite entanglement for vibrational states in a trimer molecule

Hou, Q. R., Gu, B. F. \& Chen, Y. B., Enhancement of thermo-
B29 (2015) 1540005 B29 (2015) 1550151

B29 (2015) 1550193 B29 (2015) 1550001 B29 (2015) 1550005 B29 (2015) 1550100 B29 (2015) 1550227

B29 (2015) 1542019 B29 (2015) 1550192 B29 (2015) 1550079

B29 (2015) 1540020 B29 (2015) 1542012 B29 (2015) 1550158 B29 (2015) 1550193 B29 (2015) 1550048

B29 (2015) 1550083

B29 (2015) 1550059

B29 (2015) 1540014 B29 (2015) 1550231 B29 (2015) 1542005

B29 (2015) 1542029 B29 (2015) 1550194 B29 (2015) 1542029

B29 (2015) 1550063 electric power factor in $\mathrm{CrSi}_{2}$ film via Si:B addition

Hou, X.-W., see Hou, M.

Hsu, J.-H., see Thakur, A.

Hu, B., see Wang, C.

Hu, G.-K., see Zheng, X.-Y.

Hu, J., see Ren, X.

Hu, J.-X. \& Fang, Y.-T., Magnetcontrolled surface defect modes of photonic crystal

$\mathrm{Hu}, \mathrm{X} .$, see Chen, Y.

Hu, X., see Gao, F.

Hu, X., see Liu, F.

Hu, X.-F., Wu, S.-Y., Zhang, Z.H. \& Kuang, M.-Q., Investigations on the spin Hamiltonian parameters of $\mathrm{Cu}(2)$ site in $\left(\mathrm{Tl}_{0.5} \mathrm{~Pb}_{0.5}\right) \mathrm{Sr}_{2} \mathrm{CaCu}_{2} \mathrm{O}_{7}$ superconductors

Hu, X.-F., see Kuang, M.-Q.

Hu, X.-F., see Li, G.-L.

Hu, Y. M., see Li, W. X.

Hu, Z.-D., Wang, J., Zhang, Y. \& Zhang, Y.-Q., Sudden transitions of trace distance discord of dipole-dipole coupled two qubits

Hua, D., see Yu, B.

Huang, C. \& Cao, P., The effect of reaction conditions on formation of wet precipitated calcium phosphates

Huang, G., see Chen, H.

Huang, H., see Xiao, Z.

Huang, J., see Ma, R.

Huang, K., see Li, Q.

Huang, L., see Leng, M.

Huang, R. Z., see Gao, T. F.

Huang, X. L., see Niu, X. Y.

Huang, Z., see Ouyang, X.

Huang, Z., see Zhong, K.

Hui, C., see Zhu, Z.

Hung, H.-H., see Lai, H.-H.

Hung, P. K., see Kien, P. H.

Hussain, M. K., Gao, G. Y. \& Yao, K.-L., Half-metallic properties of the new $\mathrm{Ti}_{2} \mathrm{YPb}(\mathrm{Y}=\mathrm{Co}, \mathrm{Fe})$ Heusler alloys

Hyie, K. M., see Nik Roselina, N. $\mathrm{R}$.

Ido, M., see Kurosawa, T.

Ido, M., see Shimizu, M.

Imaduddin, F., see Abd Fatah, A. Y.

Iqbal, Y., see Muhammad, R.
B29 (2015) 1550189

B29 (2015) 1550063

B29 (2015) 1550183

B29 (2015) 1550043

B29 (2015) 1550184

B29 (2015) 1550220

B29 (2015) 1550159

B29 (2015) 1550082

B29 (2015) 1550192

B29 (2015) 1542033

B29 (2015) 1542018

B29 (2015) 1542007

B29 (2015) 1542020

B29(2015) 1542032

B29 (2015) 1550138 B29(2015) 1550210

B29(2015) 1540017

B29 (2015) 1550173

B29 (2015) 1550052

B29 (2015) 1550087

B29 (2015) 1550162

B29 (2015) 1550078

B29 (2015) 1550069

B29 (2015) 1550086

B29 (2015) 1540003

B29 (2015) 1550168

B29 (2015) 1540023

B29 (2015) 1530005

B29 (2015) 1550035

B29 (2015) 1550175

B29 (2015) 1540006

B29 (2015) 1542009

B29 (2015) 1542014

B29 (2015) 1530004

B29(2015) 1550153 
Ishihara, M., Application of optimization method to the $x^{4}$ model in the Tsallis nonextensive statistics

Islam, A. K. M. A., see Ali, M. S. Izadi, M. A., see Safarpour, Gh. Jacak, L., see Gonczarek, R.

Jafari, A., Ghoranneviss, M., Boochani, A. \& Hantehzadeh, M. R., Thermoelectric properties of T-shaped graphene nanodevice

Jahangir, S., Ataie, A. \& NikkhahMoshaee, R., Effects of process control agents on the synthesis of nano-crystalline barium hexaferrite

Jani, A. R., see Jivani, A. R.

Jaramillo, J. A. P Téllez, D. A. L., Roa-Rojas, J., Vargas, C. A. P. \& Barrera, E. W., Synthesis of $\mathrm{LaYbO}_{3}$ perovskite through modified Pechini method: Structural, electric and magnetic characterization

Jarfors, A. E. W., see Bjurenstedt, A.

Jarfors, A. E. W., see Borkar, H.

Ji, Y. H. \& Wan, X. D., NonMarkovian dynamics of correlations: The composite effect of two channels and robust quantum correlation preserving by detuning

Ji, Z.., see Xie, W.

Jia, A., see Zhang, W.

Jia, S., see Yu, B.

Jia, S., see Yu, B.

Jia, Y. \& Gu, H., Phase noiseinduced double coherence resonances in a neuronal model

Jian, K. \& Wang, H., Evolution and controlling of defects during the preparation of continuous high-temperature resistant $\mathrm{SiC}(\mathrm{Al})$ fibers

Jiang, D., see Chen, Y.

Jiang, F., Yuan, H., Xie, W., Pang, Z. \& Han, S., Effect of oxygen on the magnetic property of Bis(8-hydroxyquinoline) copper $\left(\mathrm{Cu} Q_{2}\right):$ An experimental and theoretical study

Jiang, F., see Xie, W.
B29 (2015) 1450234 B29 (2015) 1550223 B29 (2015) 1550085 B29 (2015) 1550117

B29(2015) 1550133

B29 (2015) 1592002 B29 (2015) 1550132

B29 (2015) 1550217

B29 (2015) 1540011

B29 (2015) 1540015

B29 (2015) 1450249

B29 (2015) 1542042

B29 (2015) 1540026

B29 (2015) 1550210

B29 (2015) 1550031

B29(2015) 1550142

B29 (2015) 1540016 B29 (2015) 1550134

B29 (2015) 1542027 B29 (2015) 1542042
Jiang, F., see Yuan, H.

Jiang, G., see Wang, J.

Jiang, J., Gong, Y. M., Li, Y. H., Liang, G., Yang, X. S., Cheng, C. H. \& Zhao, Y., The effects of magnetization process on levitation characteristics of a superconducting bulk magnet

Jiang, J., Gong, Y. M., Wang, G., Zhou, D. J., Zhao, L. F., Zhang, Y. \& Zhao, Y., Levitation forces of a bulk YBCO superconductor in gradient varying magnetic fields

Jiang, L., see Gao, L.

Jiang, L.-H., see Zhang, Y.

Jiang, M., see Miao, R.

Jiang, P., see Wang, X. P.

Jiang, W., see You, F.

Jiang, Z., see Aljabri, A.

Jin, C. Q., see Cao, L. P.

Jin, C. Q., see Han, W.

Jin, C. Q., see Li, W. M.

Jin, C. Q., see Xing, L. Y.

Jin, W. T., see Li, L.

Jin, W., see Wang, C.

Jin, W., see $\mathrm{Xu}, \mathrm{Y}$.

Jing, L., see Guan, D.

Jivani, A. R. \& Jani, A. R., Computational investigations of mechanical and vibrational properties of quaternary semiconductor alloys $\mathrm{Mg}_{x} \mathrm{Zn}_{1-x} \mathrm{~S}_{y} \mathrm{Se}_{1-y}$

Joya, M. R., see Acosta, J. G.

Juwhari, H. K., see Mahmoud, N. $\mathrm{T}$.

Kabanov, V. V., see Watanabe, K.

Kahraman, R., see Wang, S.-J.

Kahraman, R., see Wang, Y.

Kaneyoshi, T., A transverse Ising bilayer film with an antiferromagnetic spin configuration

Kang, D., see Liu, W.

Kang, X.-W., see Zhang, Y.

Kanjouri, F., see Fathi, M. B.

Kantar, E. \& Ertaş, M., Thermodynamic quantities and phase diagrams of spin-1 Blume-Capel bilayer Ising model

B29 (2015) 1550132

B29 (2015) 1550228

B29 (2015) 1550195

B29 (2015) 1542031

B29 (2015) 1540022

B29(2015) 1540008

B29 (2015) 1550197

B29 (2015) 1542048

B29 (2015) 1550136

B29 (2015) 1550152

Karimou, M., Yessoufou, R. \& Hontinfinde, F., Critical behaviors and phase diagrams of the mixed spin-1 and spin-7/2 Blume-Capel (BC) Ising model

B29 (2015) 1550141 
Author Index

on the Bethe lattice (BL)

Kaya, A., see Tecimer, H.

Kaya, A., On the anomalous peak in the forward bias capacitance and conduction mechanism in the Au/n-4H SiC (MS) Schottky diodes (sds) in the temperature range of 140-400 $\mathrm{K}$

Kennedy, J., see Fang, F.

Khalfa, M., Chiker, F., Baki, N., Bougherara, K., Yakoubi, A., Murtaza, G., Harmel, M., AbuJafar, M. S., Bin Omran, S. \& Khenata, R., Mechanical, electronic and thermodynamic properties of full Heusler compounds $\mathrm{Fe}_{2} \mathrm{VX}(\mathrm{X}=\mathrm{Al}, \mathrm{Ga})$

Khalifeh, J. M., see Mahmoud, N. T.

Khan, M. A., see Farooq, K. Khan, M. A., see Zhou, Z.-W. Khan, Z. S., see Anwar, M. Khaniya, A., see Pantha, N. Kheirandish, F., see Refaei, A.

Khenata, R., see Boulechfar, R. Khenata, R., see El-Kelma, R. Z. Khenata, R., see Graine, R.

Khenata, R., see Khalfa, M.

Khezerlou, M. \& Goudarzi, H., Suppressed Andreev reflection and helical Andreev bound states in triplet superconductor three-dimensional topological insulator

Khordad, R., Study of strongcoupling impurity bound polaron in a quantum pseudodot

Khuntak, T., see Chainok, P.

Kien, P. H., Hung, P. K. \& Thao, N. T., Molecular dynamic simulation of Fe nanoparticles

Kim, G. S., see Ri, S. C.

Knowles, T. P. J., see Michaels, T. C. T.

Kofane, T. C., see Mboumba, M. D.

Kofane, T. C., see Mboumba, M. D.

Kofane, T. C., see Mohamadou, A. Koga, T., see Abd Fatah, A. Y.

Komorowski, P. G. \& Cottam, M. G., Localization of magnetic and electronic excitations in nanotubes with line defects

Kou, H., see Cheng, L.
B29 (2015) 1550194

B29 (2015) 1550076

B29 (2015) 1550010 B29 (2015) 1540013

B29 (2015) 1550229

B29 (2015) 1550195

B29 (2015) 1550201

B29 (2015) 1450250

B29 (2015) 1550179

B29 (2015) 1550143

B29 (2015) 1550099

B29 (2015) 1450244

B29 (2015) 1550182

B29 (2015) 1550028

B29 (2015) 1550229

B29 (2015) 1550018

B29 (2015) 1550058

B29 (2015) 1550060

B29 (2015) 1550035

B29 (2015) 1550140

B29 (2015) 1530002

B29 (2015) 1550202

B29 (2015) 1550008

B29 (2015) 1550049

B29 (2015) 1530004

B29 (2015) 1550074 B29 (2015) 1540009
Koverya, V., see Bondarenko, S.

Kreshchuk, M., A quasi-exactly solvable model: Two charges in a magnetic field subject to a non-Coulomb mutual interaction

Kruaehong, T., see Chainok, P.

Krzyzosiak, M., see Gonczarek, R.

Kuang, L., Lan, Y., Zhao, H. \& Feng, S., High-energy magnetic excitations in cuprate superconductors

Kuang, L., see Feng, S.

Kuang, M.-Q., Wu, S.-Y., Zhang, Z.-H. \& Hu, X.-F., Theoretical investigations of ${ }^{63} \mathrm{Cu}^{2+}$ orbital Knight shifts for $\mathrm{YBa}_{2} \mathrm{Cu}_{4} \mathrm{O}_{8}$

Kuang, M.-Q., see Hu, X.-F.

Kuang, M.-Q., see Li, G.-L.

Kurosawa, T., Hatta, G., Miyazaki, H., Yamaji, J., Yoshikawa, K., Nakagawa, Y., Shibata, Y., Yoshida, H., Oda, M., Ido, M., Takeyama, K. \& Momono, N., STM/STS studies for interplane disorder effects on the electronic states of the $\mathrm{Cu}-\mathrm{O}$ plane in Bi2201

Kurosawa, T., see Shimizu, M.

Kurosawa, T., see Watanabe, K.

Kushch, N. D., see Gasparov, V. A.

Kusmartsev, F. V. \& Saarela, M., Dipolar clusters and ferroelectricity in high $T_{c}$ superconductors

Kuz'menkov, L. S., see Andreev, P. A.

Kuz'menkov, L. S., see Yu. Ivanov, A.

Kuzemsky, A. L., Variational principle of Bogoliubov and generalized mean fields in manyparticle interacting systems

Kyasov, A., see Dedkov, G.

Lai, H.-H. \& Hung, H.-H., Shortranged interaction effects on the $Z_{2}$ topological phase transition: The perturbative meanfield method

Lan, Y., see Feng, S.

Lan, Y., see Kuang, L.

Lee, C. M. \& Chan, K. S., Coulomb impurity effect on
B29(2015) 1542013

B29 (2015) 1550204

B29 (2015) 1550060

B29 (2015) 1550117

B29 (2015) 1542001

B29 (2015) 1530009

B29 (2015) 1542007

B29(2015) 1542018

B29(2015) 1542020

B29 (2015) 1542009

B29 (2015) 1542014

B29 (2015) 1542031

B29 (2015) 1542012

B29 (2015) 1542002

B29(2015) 1550077

B29(2015) 1550129

B29 (2015) 1530010

B29 (2015) 1550237

B29 (2015) 1530005

B29(2015) 1530009

B29 (2015) 1542001 
electrically induced Dirac bound states in graphene

Lee, H. C., An unified approach to inelastic light scattering in Keldysh-Schwinger functional integral formalism

Lee, H. K., Structure and magnetic properties of $\left(R u_{0.9} \mathrm{Nb}_{0.1}\right) \mathrm{Sr}_{2}\left(\mathrm{Gd}_{1.34-x} \mathrm{Nd}_{x} \mathrm{Ce} \mathrm{e}_{0.66}\right) \mathrm{Cu}_{2} \mathrm{O}_{z}$ system

Lee, Y. I., Shih, C. W., Chang, W. C., Chang, H. W. \& Chen, Y. J., Inhomogeneity on texture, microstructure and magnetic properties of hot deformed $\mathrm{R}_{2} \mathrm{Fe}_{14} \mathrm{~B}$-typed magnet

Lei, M., see Zhang, H.

Leng, M., Huang, L., Li, L., Zhou, H., Cheng, J. \& Chen, X., Active semisupervised community detection based on asymmetric similarity measure

Levine, G. C., see Friedman, B. A.

Li, B., see Wei, X.

Li, D., see Bai, Y.

Li, D.-J., see Yao, Y.-B.

Li, F., see Yu, B.

Li, G.-L., Wu, S.-Y., Kuang, M.Q. \& Hu, X.-F., Theoretical studies on the Knight shifts for the tetragonal $\mathrm{Cu}^{2+}$ site in $\mathrm{HgBa}_{2} \mathrm{CuO}_{4+\delta}$

Li, H., see Che, Y.

Li, J., Tang, J. \& Zhang, Y., Design of a superconducting linear synchronous motor with YBCO coil magnet

Li, J., see Cheng, L.

Li, J., see Xie, W.

Li, L. \& Guan, J., Determining average path length and average trapping time on generalized dual dendrimer

Li, L., Guo, C. Q., Han, J. X., Yan, Y., Jin, W. T., Hao, S. J., Lin, F., Wei, K. \& Zhang, H., Role of lattice in YBCO superconductors studied by Raman spectroscopy

Li, L., Qian, L., Wang, X., Luo, S. \& Chen, X., Accurate similarity index based on activity and connectivity of node for link prediction

Li, L., see Bai, Y.
B29 (2015) 1550037

B29 (2015) 1550040

B29 (2015) 1542030

B29 (2015) 1540007 B29 (2015) 1542040

B29 (2015) 1550078

B29 (2015) 1550065

B29 (2015) 1550148

B29 (2015) 1550158

B29 (2015) 1550214

B29 (2015) 1550031

B29 (2015) 1542020 B29(2015) 1450235

B29 (2015) 1542045 B29 (2015) 1540009 B29 (2015) 1542042

B29 (2015) 1550072

B29 (2015) 1542004

B29 (2015) 1550108 B29 (2015) 1550158
Li, L., see Guo, C. Q.

$\mathrm{Li}$, L., see Leng, $\mathrm{M}$.

Li, L., see Liu, G.

Li, M., see Ren, X.

Li, N., see Liu, H.

Li, P., Zhang, Y., He, S. \& Wang, H., Analysis of opinion spreading in signed social networks under the impact of structural balance

Li, P., see Wan, K. M.

Li, Q., Huang, K. \& Chen, H., Mode competition in Er-doped fiber Bragg grating fiber laser

Li, Q., Tang, Q., Peng, T., Zhang, X., Liu, C. \& Shi, X., Molecular characteristics of $\mathrm{H}_{2} \mathrm{O}$ in hydrate/icelliquid water mixture

Li, Q., see Zhang, H.-G.

Li, Q.-C., see Wang, J.

Li, R., see Che, Y.

Li, W. M., Liu, Q. Q., Liu, Y., Feng, S. M., Wang, X. C., Jin, C. Q., Li, X. D. \& Liu, J., Synthesis and structure stability of $\mathrm{Ba}_{2} \mathrm{CuO}_{3+\delta}$ under high pressure

Li, W. M., see Cao, L. P.

Li, W. M., see Xing, L. Y.

Li, W. X., Chen, R. H., Xu, X., Hu, Y. M., Zhu, M. Y., Li, Y. \& Dou, S. X., Enhancement of critical current of $\mathrm{SiC}$ and malic acid codoped $\mathrm{MgB}_{2} / \mathrm{Fe}$ wires

Li, W.-D., see Song, C.

Li, X. D., see Li, W. M.

Li, X. T., see Liu, H.

Li, X. T., see Liu, H.

Li, X.-G., see Shu, H.-S.

Li, X.-P., see Tan, X.-H.

Li, Y. H., see Jiang, J.

Li, Y. J., see Sun, R. X.

Li, Y., Chen, T. \& Wang, X., Lamb wave band gaps in onedimensional radial phononic crystal slabs

Li, Y., see Li, W. X.

Li, Y., see Wan, M. L.

Li, Y., see Yu, B.

Li, Y., see Yu, B.

Li, Y., see Zhu, Z.

Li, Y.-T., see Zhang, H.-G.

Li, Z., see Boekema, C.

Li, Z., see Liu, G.
B29 (2015) 1542028

B29 (2015) 1550078

B29 (2015) 1550004

B29 (2015) 1550220

B29 (2015) 1542049

B29 (2015) 1550079 B29 (2015) 1550238

B29 (2015) 1550162

B29 (2015) 1550185 B29 (2015) 1550006 B29 (2015) 1542021 B29 (2015) 1450235

B29 (2015) 1542024

B29 (2015) 1542025

B29 (2015) 1542023

B29 (2015) 1542032

B29 (2015) 1450243

B29 (2015) 1542024

B29 (2015) 1542049

B29 (2015) 1542049

B29 (2015) 1550176

B29 (2015) 1550135

B29 (2015) 1542046

B29 (2015) 1542043

B29 (2015) 1550002 B29 (2015) 1542032 B29 (2015) 1550114 B29 (2015) 1550210 B29 (2015) 1550031 B29 (2015) 1540023 B29 (2015) 1550006 B29 (2015) 1542026 B29 (2015) 1550004 
Liang, G., see Jiang, J.

Liang, S.-J., see Shu, H.-S.

Liao, R., see Zhong, K.

Liao, X., see Wang, X.

Liao, Y. \& Xie, X., Independence polynomial and matching polynomial of the Koch network

Lin, F., see Guo, C. Q.

Lin, F., see Li, L.

Lin, S. P., see Tang, Z. H.

Lin, Z.-L., see Fang, Y.-T.

Liu, A. P., Yao, X. X., Wang, X., Yang, D. X. \& Zhang, X. M., Damped harmonic oscillator model for analyzing the $d y$ namic characteristics of STM system

Liu, A. P., see Sun, X. N.

Liu, A. P., see Wang, X.

Liu, A., see Chen, Q.

Liu, C., see Li, Q.

Liu, C., see Miao, R.

Liu, C.-A., see Guo, Z.-C.

Liu, C.-C., Xu, S., He, J. \& Ye, L., Unveiling the thermal entanglement in a mixed-spin XXZ model with DzyaloshinskiiMoriya interaction under a homogeneous magnetic field

Liu, F. \& Hu, X., Possible ways to synchronize phase dynamics in intrinsic Josephson junctions for terahertz radiation

Liu, G., He, J. \& Zhang, X., Compression approach of street networks considering the structural and functional features of streets

Liu, G., He, J., Luo, Z., Yang, W. \& Zhang, X., Dynamic analysis of pedestrian crossing behaviors on traffic flow at unsignalized mid-block crosswalks

Liu, G., Li, L., Guo, J. \& Li, Z., Transport optimization considering the node aggregation ability

Liu, G.-H., see Qiang, L.

Liu, H., Li, X. T., Zhou, P. B., Zhang, H., Yang, C., Ma, G. T., Liu, H., Li, X. T., Zhang, H., Yang, C., Wang, R., Chen, H., Wang, Z., Li, N. \& Zhang, L., Transmittal properties of a superconductor-ferromagnetic

B29 (2015) 1542046
B29 (2015) 1550176
B29 (2015) 1550168
B29 (2015) 1540025
B29 (2015) 1550234
B29 (2015) 1542028
B29 (2015) 1542004
B29 (2015) 1550080
B29 (2015) 1550001

B29 (2015) 1542008

B29 (2015) 1542044

B29 (2015) 1542039

B29 (2015) 1542050

B29 (2015) 1550185

B29 (2015) 1550064

B29 (2015) 1550096

B29 (2015) 1550005

B29 (2015) 1542033

B29 (2015) 1550227

B29 (2015) 1550100

B29 (2015) 1550004 B29 (2015) 1550070

\section{Love, A., see Boekema, C.}

Lu, J.-D., Liu, H.-Y., Dai, H.M., Wang, H.-J. \& Xu, B., The effects of the barrier and $\delta$-doping on the electron tunneling in a nonmagnetic heterostructure

Lu, K., see Guan, D.

Lu, M., see Wei, X.
B29 (2015) 1542049 B29 (2015) 1550170 B29 (2015) 1542049 B29 (2015) 1550165 B29 (2015) 1550178 B29 (2015) 1542024 B29 (2015) 1550135

B29 (2015) 1550116 B29 (2015) 1542025 B29 (2015) 1550019 B29 (2015) 1542024 B29 (2015) 1542023 B29 (2015) 1550176 B29 (2015) 1550178

B29 (2015) 1542048 B29 (2015) 1550212 B29 (2015) 1550203 B29 (2015) 1550026 B29 (2015) 1542011 B29 (2015) 1450247

B29 (2015) 1540029

B29 (2015) 1542024 B29 (2015) 1540025 B29 (2015) 1540003 B29 (2015) 1540027 B29 (2015) 1550027 B29 (2015) 1550128 B29 (2015) 1542025 B29 (2015) 1550066

B29 (2015) 1550125 B29 (2015) 1542026

B29 (2015) 1550165 B29 (2015) 1550104 B29(2015) 1550092 
Lu, M., see Wei, X.

Lu, P.-F., see Wei, X.-H.

Lu, T., see Guan, Q.

Lu, W., see Han, Y.

Lu, W., see Wang, Y.

Lu, X.-X., see Xiao, Z.

Lu, Y. \& Qin, Y., Influence of critical current density on magnetic force of HTSC bulk above PMR with 3D-modeling numerical solutions

Lu, Y., see Gao, L.

Lu, Y., see Qin, Y.

Luan, M., see Bai, Y.

Luo, F., see Guo, Z.-C.

Luo, M., see Guo, W. L.

Luo, S., see Li, L.

Luo, X., see Wang, J.

Luo, Z., see Liu, G.

Lyu, G.-D., Fan, C.-J., Yu, L.F., Xiu, B.-X. \& Zhang, W.M., Predicting missing links via structural similarity

Lyuksyutov, I. F., see Bang, W.

Lyuksyutov, I. F., see Bang, W.

Ma, C., see Wang, X.

Ma, D. C., see Tang, Z. H.

Ma, G. T., see Liu, H.

Ma, G. T., see Liu, W.

Ma, J., Qin, H., Song, X. \& Chu, R., Pattern selection in neuronal network driven by electric autapses with diversity in time delays

Ma, J., see Wang, C.

Ma, J., see Xing, C.

$\mathrm{Ma}$, J., see $\mathrm{Xu}, \mathrm{Y}$

Ma, R., Wan, M. P., Huang, J. \& Xie, Q., Calculation of electronic structure and mechanical properties of $\mathrm{DO}_{3}-$ $\mathrm{Fe}_{75-x} \mathrm{Si}_{25} \mathrm{Ni}_{x} \quad$ intermetallic compounds by first principles

Ma, R., see Chen, Y.

Ma, S., see Guan, Q.

Ma, S., see Miao, R.

Ma, S., Bandgap modulation and hydrogen storage with $\mathrm{Cr}$ doped BN sheets

Ma, T. \& Serota, R. A., Spectral and parametric averaging for integrable systems

Ma, X. \& Gu, W., The photoelectric characteristics of a few-layer graphene/Si Schottky
B29 (2015) 1550148

B29 (2015) 1550014

B29 (2015) 1550128

B29 (2015) 1540012

B29 (2015) 1540008

B29 (2015) 1550052

B29 (2015) 1542038

B29 (2015) 1550033

B29 (2015) 1542041

B29 (2015) 1550158

B29 (2015) 1550096

B29 (2015) 1550163

B29 (2015) 1550108

B29 (2015) 1550015

B29 (2015) 1550100

B29 (2015) 1550095

B29 (2015) 1542035

B29(2015) 1542036

B29 (2015) 1540025

B29 (2015) 1550080

B29 (2015) 1542049

B29 (2015) 1542048

B29 (2015) 1450239

B29 (2015) 1550043

B29 (2015) 1550155

B29 (2015) 1550164

B29 (2015) 1550087

B29 (2015) 1550082

B29 (2015) 1550128

B29(2015) 1550064

B29(2015) 1550160

B29 (2015) 1550109 junction solar cell

Ma, X., see Feng, S.

Ma, X., see Zhou, J.

Ma, X.-C., see Zhang, H.-G.

Macias-M, A., Rodríguez-Núñez, J. J., Bonalde, I. \& Schmidt, A. A., Analysis of experimental data for two-band superconductors at $T \approx T_{c}$ using the $G L$ theory in the presence of a selfconsistent vortex line

Maćkowiak, J., Stochastic Bose superfluid

Madan, I., see Watanabe, K.

Madani, L., Belkhiat, S., Berrag, A. \& Nemdili, S., Investigation of dielectric behavior of water and thermally aged of $\mathrm{XLPE} / \mathrm{BaTiO}_{3}$ composites in the low-frequency range

Mahapatra, S. S., see Garg, A.

Mahmoud, N. T., Mousa, A. A., Juwhari, H. K., Khalifeh, J. M. \& Abu-Jafar, M. S., Optical dispersion functions of $\mathrm{Co}_{2-x} E u_{x} V S n$ using ab-initio calculations

Majidiyan Sarmazdeh, M., see Taghavi Mendi, R.

Maksymenko, M., see Derzhko, O.

Maleki, K., Sanjabi, S. \& Alemipour, Z., AC electrodeposition of NiMn alloy nanowires in AAO template

Malik, G. P., Towards a solution of the puzzle posed by superconducting $\mathrm{SrTiO}_{3}$

Malozovsky, Y., Franklin, L., Ekuma, C. \& Bagayoko, D., Ab initio prediction of electronic, transport and bulk properties of $\mathrm{Li}_{2} \mathrm{~S}$

Mandal, A., Pan, S., Roychowdhury, A. \& Sengupta, A., PALS and DSC measurements in 8 meV electron irradiated natural rubber filled with different fillers

Mansoor, S. B. \& Yilbas, B. S., Nonequilibrium cross-plane energy transport in aluminumsilicon-aluminum wafer

Martin, G., see Danieli, C.

Massoum, N., see Belhadji, Y.
B29 (2015) 1450248

B29 (2015) 1530009

B29 (2015) 1550038

B29 (2015) 1550221

B29 (2015) 1550150

B29 (2015) 1550044

B29 (2015) 1542031

B29 (2015) 1550186 B29 (2015) 1450242

B29 (2015) 1550195

B29 (2015) 1550068

B29 (2015) 1530007

B29 (2015) 1550224

B29 (2015) 1450238

B29 (2015) 1542006

B29 (2015) 1550196

B29 (2015) 1550112 B29 (2015) 1550036 B29 (2015) 1550107 
Mastour, N., see Ridene, R.

Máté, G. \& Heermann, D. W., A generalized Potts model for confocal microscopy images

Mazlan, S. A., see Abd Fatah, A. Y.

Mboumba, M. D., Moubissi, A. B . Ekogo, T. B., Belobo Belobo, D., Ben-Bolie, G. H. \& Kofane, T. C., Stability of binary condensates with spatial modulations of quintic nonlinearities in optical lattices

Mboumba, M. D., Moubissi, A. B., Ekogo, T. B., Ben-Bolie, G. H. \& Kofane, T. C., Variational approach for two-component condensates dynamics with two- and three-body interactions and external feeding

Meng, H., Xie, W.-J. \& Zhou, W.$\mathrm{X}$., Club convergence of house prices: Evidence from China's ten key cities

Meradji, H., see Boulechfar, R.

Meradji, H., see Graine, R.

Merlin, R., A heuristic approach to the quantum measurement problem: How to distinguish particle detectors from ordinary objects

Mertelj, T., see Watanabe, K.

Meslouhi, A., see Amellal, H.

Miao, R., Yang, J., Bai, Z., Can, D., Zhang, X., Jiang, M., Liu, C., Wu, F. \& Ma, S., Firstprinciples prediction of superconductivity in $\mathrm{LiBSi}_{1-x} A l_{x}$

Michaels, T. C. T. \& Knowles, T. P. J., Kinetic theory of protein filament growth: Self-consistent methods and perturbative techniques

Mihailovic, D., see Watanabe, K.

Mirzadeh, M. \& Farjam, M., Adsorption-site dependence of electronic and magnetic properties of hydrogen impurities on bilayer graphene

Mirzaee, O., Mohamady, R., Ghasemi, A. \& Farzin, Y. A., Study of the magnetic and structural properties of $\mathrm{Al}-$ $\mathrm{Cr}$ codoped Y-type hexaferrite prepared via sol-gel auto-

\section{B29 (2015) 1550211 \\ combustion method \\ Miyazaki, H., see Kurosawa, T. \\ Mohamadou, A., Toko, D., Al- \\ B29 (2015) 1550048 \\ B29 (2015) 1530004 imTabi, C. B. \& Kofane, T. C., Modulational instability and energy localization of twisted DNA with solvent interaction}

B29 (2015) 1550008

B29 (2015) 1550202

B29 (2015) 1550181

B29 (2015) 1450244

B29 (2015) 1550028

B29 (2015) 1530011

B29 (2015) 1542031

B29 (2015) 1550232

B29 (2015) 1530002

B29 (2015) 1542031

B29 (2015) 1550198

Mohamady, R., see Mirzaee, O.

Mohammadi, A., see Firoozi, A

Mokhtari, J., see Farrokhabadi, A.

Momono, N., see Kurosawa, T.

Momono, N., see Shimizu, M.

Morigaki, K., see Hikita, H.

Morigaki, K., see Hikita, H.

Moriya, Y., see Shimizu, M.

Moubissi, A. B., see Mboumba, M. D.

Moubissi, A. B., see Mboumba, M. D.

Moulahcene, F., see Belhadji, Y.

Mounkachi, O., see Ait-Tamerd, M.

Mousa, A. A., see Mahmoud, N. $\mathrm{T}$.

Muhammad, R., Iqbal, Y. \& Rambo, C. R., Sol-gel synthesis of $\mathrm{Na}_{0.4} \mathrm{~K}_{0.6} \mathrm{Ca}_{4} \mathrm{Nb}_{5} \mathrm{O}_{17}$ microwave ceramics

Murad, M. C., see Nik Roselina, N. R.

Murayama, S., see Shimizu, M. Murtaza, G., see El-Kelma, R. Z. Murtaza, G., see Khalfa, M.

Mustafa, K., see Anwar, M.

Mwansa, P., see Song, C.

Nakagawa, Y., see Kurosawa, T.

Nakamura, S., Background story of the invention of efficient blue InGaN light emitting biodes

Naugle, D. G., see Bang, W.

Naugle, D. G., see Bang, W.

Nemdili, S., see Madani, L.

Ni, Z.-Q., see Zhang, Y.

Nik Roselina, N. R., Azizan, A., Hyie, K. M., Murad, M. C. \& Abdullah, A. H., Synthesis and characterization of $\mathrm{Ni}-\mathrm{Au}$ bimetallic nanoparticles

Nikkhah-Moshaee, R., see Hassanirad, A.

Nikkhah-Moshaee, R., see Jahangir, $\mathrm{S}$.

Niknam, E., see Safarpour, Gh. Nilkamjon, T., see Chainok, $\mathrm{P}$. Ningyu, X., see Zhihua, P.
B29(2015) 1550090

B29(2015) 1542009

B29 (2015) 1550049

B29 (2015) 1550090

B29 (2015) 1550111

B29 (2015) 1450245

B29 (2015) 1542009

B29 (2015) 1542014

B29 (2015) 1550083

B29 (2015) 1550059

B29 (2015) 1542014

B29(2015) 1550202

B29 (2015) 1550008

B29(2015) 1550107

B29(2015) 1550174

B29(2015) 1550195

B29(2015) 1550153

B29 (2015) 1540006

B29 (2015) 1542014

B29 (2015) 1550182

B29 (2015) 1550229

B29(2015) 1550179

B29 (2015) 1450243

B29 (2015) 1542009

B29 (2015) 1530016

B29 (2015) 1542035

B29 (2015) 1542036

B29 (2015) 1550186

B29(2015) 1550136

B29 (2015) 1540006

B29(2015) 1592003

B29 (2015) 1592002

B29 (2015) 1550085

B29 (2015) 1550060

B29 (2015) 1550105 
Nishimoto, N., Yoshino, K. \& Fujihara, J., Growth of $\mathrm{TiO}_{2}-$ $\mathrm{Nb}_{2} \mathrm{O}_{5}$ mixed thin films by metal-organic decomposition

Niu, X. Y., Huang, X. L., Shang, Y. F. \& Wang, X. Y., Effects of superpositions of quantum states on quantum isoenergetic cycles: Efficiency and maximum power output

Nouri, R., see Graine, R.

Novzari, M., see Safarpour, Gh.

Oda, M., see Kurosawa, T.

Oda, M., see Shimizu, M.

Oda, M., see Watanabe, K.

Omidi, A. R., see Dadsetani, M.

Omran, S. B., see El-Kelma, R. Z.

Onbaşlı, Ü., see Özdemir, Z. G.

Ostrikov, K., see Ali, M. S.

Ou-Yang, S.-L., see He, H.-L.

Ouyang, X., Cao, P., Zhang, W., Liu, Z., Huang, Z. \& Gao, W., $\mathrm{CaCu}_{3} \mathrm{Ti}_{4} \mathrm{O}_{12}-P V D F$ polymeric composites with enhanced capacitive energy density

Owens, F., see Boekema, C.

Özdemir, Z. G., Çataltepe, Ö. A. \& Onbaşlı Ü., Impedance and dielectric properties of mercury cuprate at nonsuperconducting state

Ozturk, E. \& Sokmen, I., Resonant peaks of the linear optical absorption and rectification coefficients in GaAs/GaAlAs quantum well: Combined effects of intense laser, electric and magnetic fields

Ozturk, E., Comparison of $G a_{1-x} A l_{x} A s / G a A s \quad$ and $G a_{1-x} l n_{x} A s / G a A s \quad$ quantum wells as dependent on $\mathrm{Al}$ and In concentrations under intense laser field

Pal, S. \& Bhattacharjee, J. K., Wave packet dynamics for Gross-Pitaevskii equation in one dimension: Dependence on initial conditions

Pan, H., see Cai, X.

Pan, L. Q., see Cao, L. P.

Pan, M., see Zhang, H.

Pan, S., see Mandal, A.

Pandey, R. K., A possible explana-
B29 (2015) 1550215

B29 (2015) 1550086

B29 (2015) 1550028

B29 (2015) 1550085

B29 (2015) 1542009

B29 (2015) 1542014

B29 (2015) 1542031

B29 (2015) 1550047

B29 (2015) 1550182

B29 (2015) 1550205

B29 (2015) 1550223

B29 (2015) 1550193

B29 (2015) 1540003

B29 (2015) 1542026

B29 (2015) 1550205

B29 (2015) 1550030

B29 (2015) 1550187

B29(2015) 1550216

B29 (2015) 1550236

B29 (2015) 1542025

B29 (2015) 1542040

B29 (2015) 1550196 tion of pressure dependence of critical temperature $T_{c}$ of alkali doped $C_{60}$ superconductors

B29 (2015) 1550003

Pang, X.-F., The features of infrared spectrum of bio-polymer and its theoretical investigation

Pang, Z., see Jiang, F.

Pang, Z., see Xie, W.

Pang, Z., see Yuan, $\mathrm{H}$.

Pankratov, E. L. \& Bulaeva, E. A., Optimization of manufacturing of emitter-coupled logic to decrease surface of chip

Pansari, A., Gedam, V. \& Sahoo, B. K., Built-in-polarization field effect on intrinsic and extrinsic thermal conductivities of AlN/GaN/AlN quantum well

Pantha, N., Khaniya, A. \& Adhikari, N. P., Hydrogen storage on palladium adsorbed graphene: A density functional theory study

Pareek, T. P., Quantum nonAbelian hydrodynamics: Anyonic or spin-orbital entangled liquids, nonunitarity of scattering matrix and charge fractionalization

Parisi, G., Low-temperature glassy systems: Present understanding, open problems and future developments

Parvin, R., see Ali, M. S.

Paul, S. K., see Sarkar, S.

Paul, S. K., see Sarkar, S.

Pavlov, B., see Danieli, C.

Pavuna, D., see Gasparov, V. A.

Peng, G. H., see Liu, W.

Peng, J., see Guo, X.

Peng, J., see Zhihua, $\mathrm{P}$.

Peng, T., see Li, Q.

Peter, A. J., see El Ghazi, H.

Prasad, M. S. R., Prasad, V., B. B. V. S. \& Babu, B. R., Magnetic, structural and dc electrical resistivity studies on the divalent cobalt substituted $\mathrm{Ni}-\mathrm{Zn}$ ferrite system

Prasad, M. S. R., see Babu, B. R.

Prasad, V., B. B. V. S., see Prasad, M. S. R.

Qian, L., see Li, L.

Qiang, L., Liu, G.-H. \& Tian, G.-S., Ferrimagnetic order and
B29 (2015) 1550041

B29 (2015) 1542027

B29 (2015) 1542042

B29 (2015) 1542010

B29 (2015) 1550023

B29 (2015) 1550149

B29 (2015) 1550143

B29 (2015) 1450241

B29 (2015) 1530012

B29 (2015) 1550223

B29 (2015) 1550209

B29 (2015) 1592004

B29 (2015) 1550036

B29 (2015) 1542012

B29 (2015) 1542048

B29 (2015) 1550034

B29 (2015) 1550105

B29 (2015) 1550185

B29(2015) 1550110

B29 (2015) 1550067 B29 (2015) 1550032

B29 (2015) 1550067 B29 (2015) 1550108 
Author Index

spontaneous magnetization in a mixed-spin $X X Z$ chain with single-ion anisotropy

Qin, H., see Ma, J.

Qin, J., Zhang, W., Liu, Z. \& Bai, S., Effects of polymer binder on rheological properties of silver pastes for screen printing front electrode films of solar cells

Qin, L., see Feng, S.

Qin, W., see Yang, Z.

Qin, Y. \& Lu, Y., Magnetic fields end-face effect investigation of HTS bulk over PMG with 3Dmodeling numerical method

Qin, Y., see Lu, Y.

Qin, Z., see Zhang, Y.

Qiu, K.-X., see Wang, C.-D.

Qiu, W., see Ding, M.

Qiu, Y., see Chen, Y.

Qu, L., see Gao, L.

Quang, V. X., see Tuyen, H. V.

Rach, R., see Farrokhabadi, A.

Rajesh Babu, B., see Siva Ram Prasad, M.

Rakhimov, A. \& Askerzade, I. N., Thermodynamics of noninteracting bosonic gases in cubic optical lattices versus ideal homogeneous Bose gases

Rakov, N., see Xiao, M.

Rambo, C. R., see Muhammad, R.

Ramesh, K. V., see Babu, B. R.

Ramesh, K. V., see Ramesh, M. N. V.

Ramesh, K. V., see Siva Ram Prasad, M.

Ramesh, M. N. V. \& Ramesh, K. V., Dielectric and impedance spectroscopic studies of $0.8 \mathrm{BaTiO}_{3}-0.2 \mathrm{Bi}_{0.5} \mathrm{~K}_{0.5} \mathrm{TiO}_{3}$ lead-free ceramics

Rana, A., see Anwar, M.

Rao, K. H., see Swamy, D. R. S. G.

Rao, K. S., see Swamy, D. R. S. G.

Rathnayaka, K. K. D., see Bang, W.

Rathnayaka, K. K. D., see Bang, W.

Ratreng, S., see Chainok, P.

Ravelo, B., see Thakur, A.

Rayanov, K., see Danieli, C.

Refaei, A. \& Kheirandish, F., Quantum propagator and char-

acteristic equation in the presence of a chain of $\delta$-potentials

B29 (2015) 1550070 B29 (2015) 1450239

B29 (2015) 1540027 B29 (2015) 1530009 B29 (2015) 1550207

B29 (2015) 1542041 B29 (2015) 1542038 B29 (2015) 1550050 B29 (2015) 1540031 B29 (2015) 1540028 B29 (2015) 1530003 B29 (2015) 1550033 B29 (2015) 1550235 B29 (2015) 1450245

B29 (2015) 1550101

B29 (2015) 1550123 B29 (2015) 1550146 B29 (2015) 1550153 B29 (2015) 1550032

B29 (2015) 1550119

B29 (2015) 1550101

B29 (2015) 1550119 B29 (2015) 1550179

B29 (2015) 1550218 B29 (2015) 1550218

B29 (2015) 1542035

B29 (2015) 1542036 B29 (2015) 1550060 B29 (2015) 1550183 B29 (2015) 1550036
Ren, X., Chen, S., Xie, M., Wang, S., Hu, J., Wang, S., Li, M. \& Chen, J., The foundation and study of a new type of lattice inversion potential for iridium

Rezania, H., Electronic properties of disordered zigzag carbon nanotubes

Rezania, H., The effect of boron doping on the thermal conductivity of zigzag carbon nanotubes

Rezuş, E., see Agop, M.

Ri, S. C. \& Kim, G. S., La and Co doping effects on magnetoresistance of double perovskite $\mathrm{Sr}_{2} \mathrm{FeMoO}_{6}$

Richter, J., see Derzhko, O.

Ridene, R., Mastour, N., Gamra, D. \& Bouchriha, H., Energetic behavior of excitons in hybrid organic-inorganic parabolic quantum dots and its electric field dependence

Ridene, S., see Saidi, H.

Roa-Rojas, J., see Jaramillo, J. A. $\mathrm{P}$

Robles, P., see Claro, F.

Rodríguez-Núñez, J. J., see Macias-M, A.

Rodríguez-Ponte, P., Cabra, D. \& Grandi, N., Generalized susceptibilities and Landau parameters for anisotropic Fermi liquids

Rojas, R., see Claro, F.

Roknuzzaman, M., see Ali, M. S.

Roohani, E., Arabi, H., Sarhaddi, R., Sudkhah, S. \& Shabani, A., Effect of annealing temperature on structural and magnetic properties of strontium hexaferrite nanoparticles synthesized by sol-gel auto-combustion method

Rouabhia, A., see Belarbi, W. T. Roychowdhury, A., see Mandal, A.

Ryabov, V. A., Quantum volume Saarela, M., see Kusmartsev, F. V. Sabet, M. T. M., see Bordbar, G. H.

Safarpour, Gh., Novzari, M., Izadi,
B29(2015) 1550099

B29(2015) 1550220

B29 (2015) 1550020

B29 (2015) 1550025 B29 (2015) 1550045

B29 (2015) 1550140 B29 (2015) 1530007

B29 (2015) 1550211 B29 (2015) 1550054

B29 (2015) 1550217

B29 (2015) 1450240

B29 (2015) 1550150

B29 (2015) 1550102 B29 (2015) 1450240 B29 (2015) 1550223

B29 (2015) 1550190 B29 (2015) 1550056

B29 (2015) 1550196 B29 (2015) 1550166 B29 (2015) 1542002

B29 (2015) 1550046 
M. A., Niknam, E. \& Dialameh, M., The binding energies of an off-center hydrogenic donor impurity in a GaAs/GaAlAs nanowire superlattice: Comparison between spherical and cylindrical wells

Sahin, G., see Cetinel, A.

Sahoo, B. K., see Pansari, A.

Saidi, H., Ridene, S. \& Bouchriha, $\mathrm{H}$., Hole intersubband transitions in wurtzite and zincblende strained AlGaN/GaN quantum wells and its interband interaction dependence

Sakkaris, P., see Boekema, C.

Salk, S.-H. S., see Shin, S. J.

Sangwan, K. S., see Garg, A.

Sanjabi, S., see Maleki, K.

Sarhaddi, R., see Roohani, E.

Sarkar, S., Chaudhury, R. \& Paul, S. K., The connection between vortex-like topological excitations and conventional excitations in quantum ferromagnetic spin systems on twodimensional lattice and their stability

Sarkar, S., Chaudhury, R. \& Paul, S. K., The connection between vortex-like topological excitations and conventional excitations in quantum ferromagnetic spin systems on twodimensional lattice and their stability

Satpathy, S., see Valizadeh, M. M. Schlueter, J. A., see Gasparov, V. A.

Schmidt, A. A., see Macias-M, A.

Seddik, S., see Daoud, M.

Seifeddine, S., see Bjurenstedt, A. Seifeddine, S., see Borkar, H. Sekkal, N., see Belarbi, W. T. Sengupta, A., see Mandal, A. Sereika, R., see Audzijonis, A. Serota, R. A., see Ma, T.

Shabani, A., see Roohani, E. Shakoor, R. A., see Wang, S.-J.

Shakoor, R. A., see Wang, Y.

Shaltaf amd J. Khalifeh, R., Theoretical investigation of dielectric properties of rare earth stillwellite compounds

Shan, M. \& Tian, J., Equations of

state for fluids based on hard sphere repulsion

B29 (2015) 1550089

Shang, Y. F., see Niu, X. Y.

Shao, C. \& Duan, Y., Identifying community structure in complex networks

B29 (2015) 1550085

B29 (2015) 1550093

B29 (2015) 1550149

B29 (2015) 1550054

B29 (2015) 1542026

B29 (2015) 1542003

B29 (2015) 1450242

B29 (2015) 1550224

B29 (2015) 1550190

B29 (2015) 1550209

B29 (2015) 1592004

B29 (2015) 1550219

B29 (2015) 1542012

B29 (2015) 1550150

B29 (2015) 1550124

B29 (2015) 1540011

B29 (2015) 1540015

B29 (2015) 1550056

B29 (2015) 1550196

B29 (2015) 1550167

B29 (2015) 1550109

B29 (2015) 1550190

B29 (2015) 1540022

B29 (2015) 1540008

B29 (2015) 1550154
Shariati, M., The indium oxide micro and nanopyramids: Morphology materializing and $\mathrm{H}_{2} \mathrm{~S}$ sensing properties

Sharma, A., see Thakur, A.

Sharma, P., see Thakur, A.

Shen, L., Wu, J., Liu, Z. \& Fu, G., Extremely low-frequency Lamb wave band gaps in a sandwich phononic crystal thin plate

Shen, Y., see Guan, Q.

Sheng, C.-H., Zhang, H.-D., Chen, S., Zhang, J.-C., Han, W.P. \& Long, Y.-Z., Fabrication, structural and humidity sensing properties of $\mathrm{BaTiO}_{3}$ nanofibers via electrospinning

Sherman, A., Properties of the half-filled Hubbard model investigated by the strong coupling diagram technique

Shi, D. Y. \& Wang, Z. K., Parallel lattice Boltzmann simulation of bubble rising and coalescence in viscous flows

Shi, X., see Li, Q.

Shi, X.-N., see Shu, H.-S.

Shi, Z., see Yu, S.

Shibata, Y., see Kurosawa, T.

Shih, C. W., see Lee, Y. I.

Shimizu, M., Moriya, Y., Baar, S., Momono, N., Amakai, Y., Takano, H., Murayama, S., Kurosawa, T., Oda, M. \& Ido, M., Nodal gap behavior of $\mathrm{Bi}_{2} \mathrm{Sr}_{2-x} \mathrm{Ln}_{x} \mathrm{CuO} \mathrm{O}_{6+\delta}$ ( $\mathrm{Ln}=\mathrm{La}$, Eu) investigated by specific heat measurements

Shin, S. J. \& Salk, S.-H. S., A universal scaling behavior in magnetic resonance peak in high temperature superconductivity Shu, H.-S., Wang, X.-G., Liu, R., Li, X.-G., Shi, X.-N., Liang, S.-J., Xu, L.-H. \& Dong, F.Z., Bandgap analysis of cylindrical shells of generalized phononic crystals by transfer matrix method
B29 (2015) 1550086

B29 (2015) 1550131

B29 (2015) 1550144

B29 (2015) 1550183

B29 (2015) 1550183

B29 (2015) 1550027

B29 (2015) 1550128

B29 (2015) 1550066

B29 (2015) 1550088

B29 (2015) 1550147

B29 (2015) 1550185

B29 (2015) 1550176

B29 (2015) 1550084

B29 (2015) 1542009

B29 (2015) 1540007

B29 (2015) 1542014

B29 (2015) 1542003

B29 (2015) 1550176 
Author Index

Shu, L., see Wang, J.-W.

Shu, X., see Wang, S.-J.

Shu, X., see Wang, Y.

$\mathrm{Si}, \mathrm{T} .$, Odd-even effect of melting finite polymer film on square lattice

Singh, R. P., see Chand, S.

Singh, S. K., see Chand, S.

Singru, P. M., see Garg, A.

Siva Ram Prasad, M., Rajesh Babu, B., Ramesh, K. V. \& Trinath, K., DC electrical resistivity studies and structure of $\mathrm{Ni}_{0.5} \mathrm{Zn}_{0.5} \mathrm{Cr}_{x} \mathrm{Fe}_{2-x} \mathrm{O}_{4}$ nanoferrites

Sokmen, I., see Ozturk, E.

Sokolovsky, A. I., see Gorev, V. N. Son, N. M., see Tuyen, H. V.

Song, C., Li, W.-D., Mwansa, P. \& Zhang, P., Statistical problem of ideal gas in general twodimensional regions

Song, C., see Zhang, X.-K.

Song, H., see Chen, Y.

Song, H., see Gao, F.

Song, H., see Xie, W.

Song, Q. L., see Wan, K. M.

Song, X., see Ma, J.

Song, Y. F., Zhu, Q. S., Liu, X. L., Yang, S. Y. \& Wang, Z. G., Plasmon mode coupling and depolarization shifts in AlGaAs/GaAs asymmetric step quantum wells with and without electric field

Song, Y. L., see Wan, M. L.

Stadnichuk, V., Bodrova, A. \& Brilliantov, N., Smoluchowski aggregation-fragmentation equations: Fast numerical method to find steady-state solutions

Stashans, A. \& Escobar, J., Effects of vanadium impurity on $\mathrm{TiO}_{2}$ properties

Stepanov, A. V., Information entropy of activation process: Application for low-temperature fluctuations of a myoglobin molecule

Sudkhah, S., see Roohani, E.

Sujinnapram, S., see Chainok, P.

Sun, J.-X., see Zhou, Z.-W.

Sun, R. X., Deng, Z. G., Gou, Y.

F., Li, Y. J., Zheng, J., Wang, S.
B29 (2015) 1550122
B29 (2015) 1540022
B29(2015) 1540008

B29 (2015) 1550062

B29 (2015) 1550007

B29 (2015) 1550007

B29 (2015) 1450242

B29 (2015) 1550101

B29 (2015) 1550030

B29 (2015) 1550233

B29 (2015) 1550235

B29 (2015) 1450243

B29 (2015) 1550029

B29 (2015) 1550082

B29 (2015) 1550192

B29 (2015) 1542042

B29 (2015) 1550238

B29 (2015) 1450239

B29 (2015) 1550212

B29 (2015) 1550114

B29 (2015) 1550208

B29 (2015) 1550094

B29 (2015) 1550016

B29 (2015) 1550190

B29 (2015) 1550060

B29 (2015) 1450250
Y. \& Wang, J. S., Feasibility of low-cost magnetic rail designs by integrating ferrite magnets and NdFeB magnets for HTS Maglev systems

Sun, W., see He, T.

Sun, W., see Wang, S.

Sun, X. N., Liu, A. P., Chen, Q. F. \& Wang, X., The experimental study of $\mathrm{Cr}^{6+}$ contaminated water remediation by zero-valent nano-Fe

Sun, X., see Chen, Q.

Swamy, D. R. S. G., Varma, M. C., Bharadwaj, S., Rao, K. S. \& Rao, K. H., Effect of magnesium on relaxation frequency of $\mathrm{Ni}-$ Zn nanoferrites

Tabi, C. B., see Mohamadou, A.

Taghavi Mendi, R., Majidiyan Sarmazdeh, M., Elahi, S. M., Abolhassani, M. R., Boochani, A. \& Zelati, A., Structural and electronic properties of $v$ - and codoped single-walled znont $(8,0)$ : transition from semiconducting to metallic and half-metallic states

Tai, K., see Garg, A.

Tair, F., see Belarbi, W. T.

Takano, H., see Shimizu, M.

Takeyama, K., see Kurosawa, T.

Tan, X.-H., Li, X.-P., Liu, J.-Y., Zhang, L.-H. \& Cai, J., A model for transient flow in porous media embedded with randomly distributed tree-shaped fractal networks

Tang, B., see Cheng, L.

Tang, B., see Yao, Y.-B.

Tang, H. P., see Wang, J. Z.

Tang, J., see Li, J.

Tang, Q., see Li, Q.

Tang, Q., see Yu, B.

Tang, Q., see Yu, B.

Tang, Y., see Wei, X.

Tang, Z. H., Lin, S. P., Ma, D. C. \& Wang, B., Blue and green upconversion emissions of $\mathrm{Zr}: \mathrm{Nd}: \mathrm{LiNbO}_{3}$ single crystals

Tarhan, E., see Cetinel, A.

Tay, S. L., Wang, Y., Wei, X. \& Gao, W., Effects of bismuth addition and electrodeposition
B29 (2015) 1542043

B29 (2015) 1540020

B29(2015) 1550200

B29 (2015) 1542044

B29(2015) 1542050

B29 (2015) 1550218

B29 (2015) 1550049

B29 (2015) 1550068

B29 (2015) 1450242

B29 (2015) 1550056

B29 (2015) 1542014

B29 (2015) 1542009

B29 (2015) 1550135

B29 (2015) 1540009

B29(2015) 1550214

B29 (2015) 1540002

B29 (2015) 1542045

B29 (2015) 1550185

B29 (2015) 1550210

B29 (2015) 1550031

B29 (2015) 1540021

B29 (2015) 1550080

B29 (2015) 1550093 
processing on Zn-based coatings

Tay, S., see Wang, S.-J.

Tecimer, H., Vural, Ö., Kaya, A. \& Altındal, Ş.,Current-transport mechanism in Au/V-doped PVC $+T C N Q / p$-Si structures

Téllez, D. A. L., see Jaramillo, J. A. $P$

Teizer, W., see Bang, W.

Teizer, W., see Bang, W.

Thakur, A., Sharma, P., Thakur, P., Sharma, A., Thakur, A., Ravelo, B. \& Hsu, J.-H., Effect of high spin $\mathrm{Mn}^{2+} / \mathrm{Mn}^{3+}$ ions on microstructural, optical, magnetic and electrical properties of hydrothermally prepared $\mathrm{Ni}-$ $M g$ nanoferrites

Thakur, A., see Thakur, A.

Thakur, P., see Thakur, A.

Thao, N. T., see Kien, P. H.

Tian, G.-S., see Qiang, L.

Tian, J., see Shan, M.

Tian, J., see Yi, H.

Tian, M., see Wan, M. L.

Tian, Q., see Wu, Z.

Tian, W.-C., Wang, Z. \& Yao, D.-X., Fidelity susceptibility in multiband one-dimensional topological superconducting system

Tian, X., see Zhang, X.-K.

Tiyasri, S., see Chainok, P.

Toda, Y., see Watanabe, K.

Toko, D., see Mohamadou, A.

Tran, L. Q. N., Yuan, X. W., Bhattacharyya, D., Fuentes, C., Van Vuure, A. W. \& Verpoest, I., Fiber-matrix interfacial adhesion in natural fiber composites

Trinath, K., see Siva Ram Prasad, M.

Tronin, V. N., see Borman, V. D.

Tsuchiya, S., see Watanabe, K.

Tu, M., see Liu, Y.

Tuan, D. A., Tung, V. T., Chuong, T. V. \& Hong, L. V., Properties of lead-free BZT-BCT ceramics synthesized using nanostructured $\mathrm{ZnO}$ as a sintering aid

Tung, V. T., see Tuan, D. A.

Tuyen, H. V., Son, N. M. \& Quang, V. X., Structural behavior and
Judd-Ofelt intensity parameter of $\mathrm{Sr}_{3} \mathrm{~B}_{2} \mathrm{O}_{6}: \mathrm{Eu}^{3+}$ phosphor

B29 (2015) 1550235

Udomsamuthirun, P., see Chainok, P.

B29 (2015) 1550060

Valizadeh, M. M. \& Satpathy, S., RKKY interaction for the spinpolarized electron gas

B29(2015) 1550076

B29 (2015) 1550217

B29 (2015) 1542035

B29 (2015) 1542036

Van Vuure, A. W., see Tran, L. Q. $\mathrm{N}$.

Vargas, C. A. P., see Jaramillo, J. A. $P$

Varma, M. C., see Swamy, D. R. S. G.

Venuti, L. C. \& Zanardi, P., Theory of temporal fluctuations in isolated quantum systems

Verpoest, I., see Tran, L. Q. N.

Vijayaraghavan, V., see Garg, A.

B29 (2015) 1550183

B29 (2015) 1550183

B29 (2015) 1550183

B29 (2015) 1550035

B29 (2015) 1550070

B29 (2015) 1550089

B29 (2015) 1550156

B29 (2015) 1550114

B29 (2015) 1550213

B29 (2015) 1542034 B29 (2015) 1550029 B29 (2015) 1550060 B29 (2015) 1542031 B29 (2015) 1550049

B29 (2015) 1540018

B29 (2015) 1550101

B29 (2015) 1550097

B29 (2015) 1542031

B29 (2015) 1540029

B29 (2015) 1550231 B29 (2015) 1550231

Vural, Ö., see Tecimer, H.

Wan, K. M., Zhang, Y. J., Li, P., Wang, G., Xiang, J., Ding, B. F., Alameh, K. \& Song, Q. L., The effect of an external electric field on thermallydeposited thin CdS/CdTe-based solar cells

Wan, L., see Wei, X.-H.

Wan, M. L., Yuan, S. Q., Song, Y. L., Li, Y., Tian, M. \& Zhou, F. Q., Magnetic-coupling induced transparency in a planar terahertz metamaterial

Wan, M. P., see Ma, R.

Wan, X. D., see Ji, Y. H.

Wang, B., see Tang, Z. H.

Wang, C., Ma, J., Hu, B. \& Jin, W., Formation of multi-armed spiral waves in neuronal network induced by adjusting ion channel conductance

B29 (2015) 1550238 B29 (2015) 1550014

B29 (2015) 1550114 B29 (2015) 1550087 B29 (2015) 1450249 B29 (2015) 1550080

Wang, C.-D., Qiu, K.-X., Chen, M. \& Cai, X.-J., Machinability of drilling T700/LT-03A carbon fiber reinforced plastic (CFRP) composite laminates using candle stick drill and multi-facet drill

B29 (2015) 1540031

Wang, C.-Y., Anomalous barrier escaping in an externally modulated environment of systemreservoir coupling

B29 (2015) 1550012

B29 (2015) 1540024

B29 (2015) 1540034

B29 (2015) 1550033

B29 (2015) 1542048 
Wang, F., see Yuan, H.

Wang, G., see Jiang, J.

Wang, G., see Wan, K. M.

Wang, G.-J., see Yan, X.-G.

Wang, H., see Jian, K.

Wang, H., see Li, $\mathrm{P}$.

Wang, H.-J., see Lu, J.-D.

Wang, J. S., see Liu, W.

Wang, J. S., see Sun, R. X.

Wang, J. Z., Ao, Q. B., Tang, H. P. \& Bao, T. F., Effect of characterization of porous metal fiber media on sound absorption coefficient

Wang, J., Xu, J. J., Yang, Y., Wang, X. J., Luo, X., Zhang, L. \& Jiang, G., Simulations on the gelling process of particle suspension systems for in-situ preparing porous materials in a capillary

Wang, J., Zhou, H.-F., Li, Q.C., Dong, H.-N., Investigations on thermodynamic properties of the three sub-lattice spin frustrated chain

Wang, J., see Che, Y.

Wang, J., see Hu, Z.-D.

Wang, J., see Wei, X.

Wang, J., see Wei, X.

Wang, J.-W. \& Shu, L., Bidirectional quantum controlled teleportation of qudit state via partially entangled GHZ-type states

Wang, K., see Chen, Y.

Wang, L. C., see Farooq, K.

Wang, L. F., see Gao, T. F.

Wang, L. \& Chung, S. G., Entanglement perturbation theory for infinite quasi-1D quantum systems

Wang, M., see Zha, D.

Wang, Q., see Zhang, S. T.

Wang, R., Zhou, J.-N., Liu, X.-M. \& Xiao, H., Transport and efficiency of Brownian particles in confined narrow channels with a periodic driving force

Wang, R., see Liu, H.

Wang, R.-N., see Yang, M.

Wang, S. Y., see Sun, R. X.

Wang, S., Sun, W. \& Zheng, S., Calculations of first passage time of delayed tree-like net-

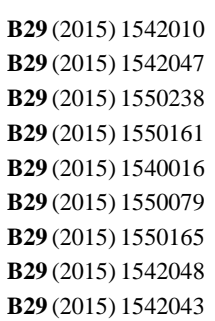

B29 (2015) 1540002

B29 (2015) 1550015

B29 (2015) 1542021

B29 (2015) 1450235

B29 (2015) 1550138

B29 (2015) 1550092

B29 (2015) 1550148

B29 (2015) 1550122

B29 (2015) 1550082

B29 (2015) 1550201

B29 (2015) 1550069

B29 (2015) 1550042

B29 (2015) 1550061

B29 (2015) 1550178

B29 (2015) 1550026

B29 (2015) 1542049

B29 (2015) 1550073

B29 (2015) 1542043 works

Wang, S., see Cui, H.

Wang, S., see Ren, X.

Wang, S., see Ren, X.

Wang, S.-J., Wang, Y., Shu, X., Tay, S., Gao, W., Shakoor, R. A. \& Kahraman, R., Preparation and property of duplex $\mathrm{Ni}-\mathrm{B}-$ $\mathrm{TiO}_{2} / \mathrm{Ni}$ nano-composite coatings

Wang, S.-S. \& Zha, G.-Q., Geometric effect on the vortex configuration and motion in mesoscopic superconducting cylinders

Wang, X. C., see Cao, L. P.

Wang, X. C., see Han, W.

Wang, X. C., see Li, W. M.

Wang, X. C., see Xing, L. Y.

Wang, X. J., see Wang, J.

Wang, X. P., Jiang, P., Chen, T. N. \& Bao, K., Lamb wave band structure in phononic crystal composed of multiple square stubs symmetric or antisymmetric deposited on a homogeneous plate

Wang, X. Y., see Niu, X. Y.

Wang, X., Liao, X., Ma, C., Zhang, S., Liu, Y. \& Chen, H., Effects of chemical composition on the corrosion behavior of A7NO1ST5 Al alloys

Wang, X., Liu, A. P. \& Yang, X. H., System design and new applications for atomic force microscope based on tunneling

Wang, X., see Liu, A. P.

Wang, X., see Li, L.

Wang, X., see Li, Y.

Wang, X., see Sun, X. N.

Wang, X., see Xiong, J.

Wang, X.-G., see Shu, H.-S.

Wang, X.-X., see Xu, X.-L.

Wang, Y., Shu, X., Gao, W., Shakoor, R. A., Kahraman, R., Yan, P., Lu, W. \& Yan, B., Microstructure and properties of $\mathrm{Ni}-\mathrm{Co}-\mathrm{TiO}_{2}$ composite coatings fabricated by electroplating

Wang, Y., see He, T.

Wang, Y., see Tay, S. L.

Wang, Y., see Wang, S.-J.

Wang, Z. G., see Song, Y. F.
B29 (2015) 1550200

B29 (2015) 1550203

B29 (2015) 1550220

B29(2015) 1550220

B29 (2015) 1540022

B29 (2015) 1550009

B29 (2015) 1542025

B29 (2015) 1550019

B29 (2015) 1542024

B29 (2015) 1542023

B29 (2015) 1550015

B29 (2015) 1550106 B29 (2015) 1550086

B29 (2015) 1540025

B29 (2015) 1542039

B29 (2015) 1542008

B29 (2015) 1550108

B29 (2015) 1550002

B29 (2015) 1542044

B29 (2015) 1550191

B29(2015) 1550176

B29 (2015) 1550169

B29 (2015) 1540008

B29 (2015) 1540020

B29 (2015) 1540010

B29 (2015) 1540022

B29 (2015) 1550212 
Wang, Z. K., see Shi, D. Y.

Wang, Z. S., see Cai, X.

Wang, Z., see Chen, Y.

Wang, Z., see Chi, Z.

Wang, Z., see Guo, X.

Wang, Z., see Liu, H.

Wang, Z., see Tian, W.-C.

Watanabe, K., Tsuchiya, S., Toda, Y., Kurosawa, T., Oda, M., Mertelj, T., Madan, I., Kabanov, V. V. \& Mihailovic, D., Time-resolved polarimetry for photoexcited $Q P$ dynamics in Bi2212

Wei, D., see Aljabri, A.

Wei, K., see Guo, C. Q.

Wei, K., see Li, L.

Wei, X., Li, B., Lu, M., Yi, G. \& Wang, J., Modulation of spike coding by subthreshold extracellular electric fields and neuronal morphology

Wei, X., Yang, Z., Tang, Y. \& Gao, W., Influence of $\mathrm{Al}_{2} \mathrm{O}_{3}$ sol concentration on the microstructure and mechanical properties of $\mathrm{Cu}-\mathrm{Al}_{2} \mathrm{O}_{3}$ composite coatings

Wei, X., Yin, X., Lu, M., Yi, G. \& Wang, J., Dependence of sinusoidal electric field effect on neuronal morphological properties

Wei, X., see Che, Y.

Wei, X., see Tay, S. L.

Wei, X.-H., Gao, T., Wan, L. \& Lu, P.-F., First-principles study on the electronic structure and optical properties of GaAs nanowires

Wen, G., see Hayat, M. D.

Wong, C. H., see Garg, A.

Wongphakdee, W., see Chainok, $\mathrm{P}$.

$\mathrm{Wu}, \mathrm{C} . \mathrm{X} .$, Traveling wave solution of higher-order traffic flow model with discontinuous fundamental diagram

Wu, F., see Gao, F.

Wu, F., see Miao, R.

Wu, H., Cheng, L. F., Fan, S. W., Yuan, X. W. \& Bhattacharyya, D., The effects of pre-oxidation heating rate on bio-based carbon fibers and its surface re-
B29 (2015) 1550147

B29(2015) 1550236

B29 (2015) 1530003

B29 (2015) 1550199

B29 (2015) 1550034

B29 (2015) 1542049

B29 (2015) 1542034

B29 (2015) 1542031

B29 (2015) 1540032

B29 (2015) 1542028

B29 (2015) 1542004

B29(2015) 1550148

B29 (2015) 1540021

B29 (2015) 1550092

B29 (2015) 1450235

B29 (2015) 1540010

B29 (2015) 1550014

B29 (2015) 1540005

B29 (2015) 1450242

B29 (2015) 1550060

B29 (2015) 1550137 B29 (2015) 1550192 B29(2015) 1550064 pair

Wu, J. H., see Gao, N.

Wu, J. H., see Guan, D.

Wu, J., see Shen, L.

Wu, J., see Zha, D.

Wu, R., see Chen, H.

Wu, S. Y., see Ding, C. C.

Wu, S. Y., see Zhang, L. J.

Wu, S.-Y, see Xu, Y.-Q.

Wu, S.-Y., see He, J.-J.

Wu, S.-Y., see Hu, X.-F.

Wu, S.-Y., see Kuang, M.-Q.

Wu, S.-Y., see Li, G.-L.

Wu, X. S., see Zhang, G. P.

Wu, X.-L., Zhou, X.-L. \& Chang, J., Structural stability and elastic properties of $\mathrm{WB}_{4}$ under high pressure

Wu, Y., Duan, G. \& Zhao, X., Effects of magnetic field intensity on carbon diffusion coefficient in pure iron in $\gamma$-Fe temperature region

Wu, Z., Chen, L. \& Tian, Q., Exciton binding energies in GaAs films on $A l_{x} G a_{1-x}$ As substrates Xi, X.-Q., see Chen, W.-X.

Xia, Y.-Q., see Hou, M.

Xiang, J., see Wan, K. M.

Xiao, C., see Guan, Q.

Xiao, H., see Wang, R.

Xiao, M. \& Rakov, N., Size and temperature dependent plasmons of quantum particles

Xiao, Z., Huang, H. \& Lu, X.$\mathrm{X} .$, Resonant tunneling dynamics and the related tunneling time

Xiaogui, Z., see Zhihua, P.

Xie, C., see Yan, X.-G.

Xie, J., see Feng, P.

Xie, K., see Xu, J.

Xie, L., see Chen, Y.

Xie, L., see Zhang, H.-G.

Xie, L., see Zhang, H.-G.

Xie, M., see Ren, X.

Xie, Q., see Ma, R.

Xie, W., Pang, Z., Fan, J., Song, H., Jiang, F., Yuan, H., Li, J., Ji, Z.. \& Han, S., Structural properties of $\mathrm{Alq}_{3}$ nanocrystals prepared by physical vapor deposition and facile solution method Xie, W., see Jiang, F.

Xie, W., see Yuan, H.
B29 (2015) 1540019

B29 (2015) 1550017

B29 (2015) 1550104

B29 (2015) 1550027

B29 (2015) 1550061

B29 (2015) 1550173

B29 (2015) 1542015

B29 (2015) 1542017

B29 (2015) 1542016

B29 (2015) 1542019

B29 (2015) 1542018

B29 (2015) 1542007

B29 (2015) 1542020

B29 (2015) 1550115

B29 (2015) 1550103

B29 (2015) 1540001

B29 (2015) 1550213 B29 (2015) 1550098 B29 (2015) 1550063 B29 (2015) 1550238 B29 (2015) 1550128 B29 (2015) 1550026

B29 (2015) 1550146

B29 (2015) 1550052 B29 (2015) 1550105 B29 (2015) 1550161 B29 (2015) 1550055 B29 (2015) 1540030 B29 (2015) 1550134 B29 (2015) 1550221 B29 (2015) 1550006 B29 (2015) 1550220 B29 (2015) 1550087

B29 (2015) 1542042 B29 (2015) 1542027 B29 (2015) 1542010 
Xie, W.-J., see Meng, H.

Xie, X., see Liao, Y.

Xie, Y.-X., see Chen, W.-X.

Xing, C., Yang, L. \& Ma, J., A deterministic pseudo-fractal networks with time-delay

Xing, L. Y., Wang, X. C., Deng, Z., Zhang, S. J., Feng, S. M., Li, W. M., Liu, Q. Q. \& Jin, C. Q., The crossover from metal to semiconductor in Cu-doped LiFeAs system

Xiong, J., Chen, T., Wang, X. \& $\mathrm{Zhu}, \mathrm{J}$., Design and assessment of an acoustic ground cloak with layered structure

Xiong, J., see Yang, T.

Xiu, B.-X., see Lyu, G.-D.

$\mathrm{Xu}, \mathrm{B} .$, see $\mathrm{Lu}$, J.-D.

Xu, G., see Zhong, K.

$\mathrm{Xu}$, J. J., see Wang, J.

$\mathrm{Xu}, \mathrm{J}$. \& Xie, K., Thermal radiation characteristics of silicon inverse opal in mid infrared range

$\mathrm{Xu}$, J., Lazy states, discordant states and entangled states for 2-qubit systems

Xu, L.-H., see Shu, H.-S.

$\mathrm{Xu}, \mathrm{S} .$, see Liu, C.-C.

$\mathrm{Xu}, \mathrm{W}$. , see Zhou, J.

Xu, X., see Li, W. X.

Xu, X.-L., Wang, X.-X. \& Fan, H.Y., Energy variation of mesoscopic $L-C$ electric circuit in external electromagnetic field

Xu, Y. \& Zhang, C., Topological Fulde-Ferrell superfluids of a spin-orbit coupled Fermi gas

Xu, Y., Jin, W. \& Ma, J., Emergence and robustness of target waves in a neuronal network

Xu, Y.-Q., Wu, S.-YZhang, Z.H. \& Dong, H.-N., Theoretical investigations of the $\mathrm{g}$ factors of orthorhombic $\mathrm{Cu}^{2+}$ site in oxycarbonate phase $\mathrm{YBa}_{2} \mathrm{Cu}_{2.95}\left(\mathrm{CO}_{3}\right)_{0.35} \mathrm{O}_{6.6}$

Xu, Z., Hodgson, M. A. \& Cao, P., Microstructure and degradation behavior of forged $\mathrm{Fe}-\mathrm{Mn}$ Si alloys

Xue, X., see Cheng, L.

Ya. Kezerashvili, R., see Grigorenko, I.

\begin{tabular}{l|ll} 
B29(2015) 1550181 & Yagubskii, E. B., see Gasparov, V. & \\
B29(2015) 1550234 & A. & B29 (2015) 1542012 \\
B29(2015) 1550098 & Yakoubi, A., see Khalfa, M. & B29 (2015) 1550229 \\
& Yamaji, J., see Kurosawa, T. & B29 (2015) 1542009 \\
& Yan, B., see Wang, Y. & B29(2015) 1540008 \\
B29(2015) 1550155 & Yan, B., see Yan, P. & B29(2015) 1540024 \\
& Yan, B., see Yan, P. & B29(2015) 1540034
\end{tabular}

B29 (2015) 1542023

B29 (2015) 1550191

B29 (2015) 1540033

B29 (2015) 1550095

B29 (2015) 1550165

B29 (2015) 1550168

B29 (2015) 1550015

B29 (2015) 1540030

B29 (2015) 1550121

B29 (2015) 1550176

B29 (2015) 1550005

B29 (2015) 1550038

B29 (2015) 1542032

B29 (2015) 1550169

Yan, P., Wang, D. \& Yan, B., Microstructure and wear property of spray formed high leaded bronze

Yan, P., Wang, D. \& Yan, B., Ultrasonic gas alloy atomization under near-zero aspiration pressure

Yan, P., see Wang, Y.

Yan, X.-G., Xie, C. \& Wang, G.J., Stock market network's topological stability: Evidence from planar maximally filtered graph and minimal spanning tree

Yan, Y., see Guo, C. Q.

Yan, Y., see Li, L.

Yanfeng, P., see Zhihua, P.

Yang, C., see Liu, H.

Yang, C., see Liu, H.

Yang, D. X., see Liu, A. P.

Yang, F. \& Gabbitas, B., Effect of heat treatment on microstructures and mechanical properties of a Ti-6Al-4V alloy rod prepared by powder compact extrusion

Yang, G.-R., see Dong, H.-N.

Yang, J., see Miao, R.

Yang, L., see Xing, C.

Yang, M. \& Wang, R.-N., Topological insulator in telluriumbased perovskites

Yang, S. Y., see Song, Y. F.

Yang, T., Xiong, J., Chen, H. \&

B29 (2015) $1550164 \quad$ Chen, Y., Modeling of weld bead geometry for rapid manufacturing by robotic GMAW

Yang, T., see Che, Y.

Yang, W., see Liu, G.

Yang, X. F., see Liu, W.

B29 (2015) 1542016

Yang, X. H., see Wang, X.

Yang, X. S., see Jiang, J.

Yang, X., see Cui, H.

Yang, X., see Han, Y.

B29 (2015) 1540014

B29 (2015) 1540009

Yang, Y., see Wang, J.

Yang, Z., Gao, M. \& Qin, W., Transfer of high-dimensional

B29 (2015) 1550188 quantum state through an
B29 (2015) 1540024

B29 (2015) 1540034 B29 (2015) 1540008

B29 (2015) 1550161

B29(2015) 1542028

B29 (2015) 1542004

B29 (2015) 1550105

B29 (2015) 1542049

B29 (2015) 1542049

B29 (2015) 1542008

B29 (2015) 1540004

B29 (2015) 1542022

B29 (2015) 1550064

B29 (2015) 1550155

B29 (2015) 1550073

B29 (2015) 1550212

B29 (2015) 1540033

B29 (2015) 1450235

B29 (2015) 1550100

B29 (2015) 1542048

B29 (2015) 1542039

B29 (2015) 1542046

B29 (2015) 1550203

B29 (2015) 1540012

B29(2015) 1550015 


\section{$X X Z$-Heisenberg quantum spin chain}

Yang, Z., see Wei, X.

Yao, D.-X., see Tian, W.-C.

Yao, J., see Cao, X.

Yao, K.-L., see Hussain, M. K.

Yao, X. X., see Liu, A. P.

Yao, Y.-B., Li, D.-J. \& Tang, B., Semiclassical intrinsic localized modes in the $\beta$-FermiPasta-Ulam lattice model with first- and second-nearest neighbor interactions

Ye, L., see Liu, C.-C.

Yessoufou, R., see Karimou, M.

Yi, G., see Wei, X.

Yi, G., see Wei, X.

Yi, H. \& Tian, J., New generalized corresponding states correlation for surface tension of normal saturated liquids

Yi, X. X., see Farooq, K.

Yilbas, B. S., see Mansoor, S. B.

Yildirim, E. K., 2D quasi-planar or $3 D$ structures? A comparison between $\mathrm{CrB}_{n}(n=2-10)$ wheel-like clusters and their corresponding $3 D$ pyramidal clusters, and their hydrogen storage capability

Yıldırım, M., see Gökçen, M.

Yıldız, D. E., see Çetinkaya, H. G. Yin, J., Zhang, S., Zhang, H. W. \& Chen, B. S., Band structure and transmission characteristics of complex phononic crystals by multi-level substructure scheme

Yin, S. \& Chen, Y., Influence of next-nearest neighbor interaction on low temperature properties of the spin-1/2 onedimensional ferromagnetic $X Y$ model

Yin, X., see Wei, X.

Yoshida, H., see Kurosawa, T.

Yoshikawa, K., see Kurosawa, T.

Yoshino, K., see Nishimoto, N.

You, F., Chen, J., Fan, H. \& Jiang, W., Evolution of the number state in laser process

Yu, B., Li, Y., Tang, Q., Hua, D. \& Jia, S., Correlated electron dynamics of nonsequential double ionization by few-cycle laser pulses with different time durations

B29(2015) 1550210

Yu, B., Tang, Q., Li, Y., Li, F. \& $\mathrm{Jia}, \mathrm{S}$. , Role of $e-e$ repulsion interaction in nonsequential double ionization of Xe by linearly and elliptically polarized laser pulses

Yu, J.-X., Liu, X.-Y., Zhang, L.-Y., Cheng, Y. \& Chen, X.-R., Firstprinciples quantum transport in $\mathrm{S}_{3}$ clusters

B29(2015) 1550214

B29 (2015) 1550005

B29 (2015) 1550194

B29 (2015) 1550092

B29 (2015) 1550148

B29 (2015) 1550156

B29 (2015) 1550201

B29 (2015) 1550112

Yu, L., see Gao, N.

Yu, L.-F., see Lyu, G.-D.

Yu, S. \& Shi, Z., Fuel consumptions and exhaust emissions induced by cooperative adaptive cruise control strategies

Yu. Ivanov, A. \& Kuz'menkov, L. S., Influence of quantum energy equation on electronic plasma oscillations

Yuan, H., Wang, F., Jiang, F., Xie, W., Zhang, X., Pang, Z. \& Han, S., Cu-induced spin polarization in tris(8-hydroxyquinoline) aluminum

Yuan, H., see Jiang, F.

Yuan, H., see Xie, W.

B29 (2015) 1550172

B29 (2015) 1550120

B29 (2015) 1450237

Yuan, S. Q., see Wan, M. L.

Yuan, X. W., see Tran, L. Q. N.

Yuan, X. W., see Wu, H.

Yücedağ, İ., Ersöz, G., Gümüş, A. \& Altındal, Ş., Dielectric properties and electric modulus of Au/PPy/n-Si (MPS) type Schottky barrier diodes (SBDS) as a function of frequency and applied bias voltage

Žaltauskas, R., see Audzijonis, A.

Zamzuri, H., see Abd Fatah, A. Y.

Zanardi, P., see Venuti, L. C.

Zandi Goharrizi, A., Alahyarizadeh, Gh., Hassan, Z. \& Abu Hassan, H., The influence of quaternary electron blocking layer on the performance characteristics of intracavity-contacted oxideconfined InGaN-based vertical cavity surface emitting lasers

B29(2015) 1550139

Zaster, S. \& Bittner, E. R., Role of dark excitations in the nonequilibrium condensation of exciton polaritons in optically-pumped
B29 (2015) 1550031

B29 (2015) 1450247

B29 (2015) 1550017

B29 (2015) 1550095

B29 (2015) 1550084

B29 (2015) 1550129

B29 (2015) 1542010

B29 (2015) 1542027

B29 (2015) 1542042

B29 (2015) 1550114

B29 (2015) 1540018

B29(2015) 1540019

B29 (2015) 1550075

B29 (2015) 1550167

B29 (2015) 1530004

B29 (2015) 1530008

B29 (2015) 1550230 
organic single crystal microcavities

Zeinali, M., see Abd Fatah, A. Y. Zelati, A., see Taghavi Mendi, R. Zeng, W., see Han, Y.

Zha, D., Chen, C., Wu, J. \& Wang, M., Effect of $S W$ defect on structural and transport properties of silicene nanoribbons

Zha, G.-Q., see Wang, S.-S.

Zhang, C., see Xu, Y.

Zhang, G. P., Zhu, H. P., Bai, Y. H., Bonacum, J., Wu, X. S. \& George, T. F., Imaging superatomic molecular orbitals in a $\mathrm{C}_{60}$ molecule through four 800nm photons

Zhang, H. W., see Yin, J.

Zhang, H., Zhao, Y., Zhang, Y., Pan, M. \& Lei, M., $\mathrm{SmBa}_{2} \mathrm{Cu}_{3} \mathrm{O}_{7-\delta}$ superconducting layer prepared on $\mathrm{NiO}$ buffer layer through fluorinefree chemical method

Zhang, H., see Guo, C. Q.

Zhang, H., see Liu, H.

Zhang, H., see Liu, H.

Zhang, H., see Li, L.

Zhang, H.-D., see Sheng, C.-H.

Zhang, H.-G., Li, Y.-T., Xie, L., Dong, X.-G. \& Li, Q., Annealing temperature dependence of local atomic and electronic structure of polycrystalline $\mathrm{La}_{0.5} \mathrm{Sr}_{0.5} \mathrm{MnO}_{3}$

Zhang, H.-G., Ma, X.-C. \& Xie, $\mathrm{L}$., The structural and magnetic properties of Sr-doped multiferroic $\mathrm{CaMn}_{7} \mathrm{O}_{12}$

Zhang, J., see Zhong, K.

Zhang, J.-C., see Sheng, C.-H.

Zhang, L. J., Wu, S. Y., Ding, C. C. \& Cheng, Y. K., Theoretical studies of the anisotropic $\mathrm{g}$ factors for deoxygenated Y123

Zhang, L. J., see Ding, C. C.

Zhang, L. L., see Guo, W. L.

Zhang, L., see Liu, H.

Zhang, L., see Wang, J.

Zhang, L., Nonlinear refractive index change and optical rectification in a GaN-based step quantum wells with strong built-in electric field

Zhang, L.-H., see Tan, X.-H.
B29 (2015) 1550157

B29 (2015) 1530004

B29 (2015) 1550068

B29 (2015) 1540012

B29 (2015) 1550061 B29 (2015) 1550009 B29 (2015) 1530001

B29 (2015) 1550115

B29 (2015) 1550013

B29 (2015) 1542040

B29 (2015) 1542028

B29 (2015) 1542049

B29 (2015) 1542049

B29 (2015) 1542004

B29 (2015) 1550066

B29 (2015) 1550006

B29 (2015) 1550221

B29 (2015) 1550168

B29 (2015) 1550066

B29 (2015) 1542017

B29 (2015) 1542015

B29 (2015) 1550163

B29 (2015) 1542049

B29 (2015) 1550015

B29 (2015) 1550024 B29 (2015) 1550135
Zhang, L.-L., see Zheng, X.-Y.

Zhang, L.-Y., see Yu, J.-X.

Zhang, M., see Zhu, Z.

Zhang, P., see Song, C.

Zhang, Q., see Chen, Q.

Zhang, S. J., see Xing, L. Y.

Zhang, S. T., Wang, Q., Liu, T. T. \& Liu, J. J., Controlling crystallization process and thermal stability of a binary $\mathrm{Cu}-\mathrm{Zr}$ bulk metallic glass via minor element addition

Zhang, S., see Wang, $\mathrm{X}$.

Zhang, S., see Yin, J.

Zhang, W., Chen, X., Jia, A. \& Bai, S., Highpermittivity microwave dielectric ceramics based on $(1-x)\left(\mathrm{Li}_{1 / 2} \mathrm{Nd}_{1 / 2}\right) \mathrm{TiO}_{3}-$ $x \mathrm{CaTiO}_{3}$

Zhang, W., see Ding, M.

Zhang, W., see Ouyang, X.

Zhang, W., see Qin, J.

Zhang, W.-M., see Lyu, G.-D.

Zhang, X. M., see Liu, A. P.

Zhang, X. R., see Guo, W. L.

Zhang, X., see Liu, G.

Zhang, X., see Liu, G.

Zhang, X., see Li, Q.

Zhang, X., see Miao, R.

Zhang, X., see Yuan, $\mathrm{H}$.

Zhang, X.-K., Fei, S., Song, C., Tian, X. \& Ao, Y.-Y., Label propagation algorithm based on local cycles for community detection

Zhang, X.-L., see Guo, Z.-C.

Zhang, X.-R., see Zheng, X.-Y.

Zhang, Y. J., see Wan, K. M.

Zhang, Y. \& Qin, Z., Spinscattering at the interface of normal and spin-orbit interaction heterostructure

Zhang, Y., Ni, Z.-Q., Jiang, L.-H., Han, L. \& Kang, X.-W., Study of the bending vibration characteristic of phononic crystals beam-foundation structures by Timoshenko beam theory

Zhang, Y., see Hu, Z.-D.

Zhang, Y., see Jiang, J.

Zhang, Y., see Li, J.

Zhang, Y., see Li, P.

Zhang, Y., see Zhang, H.

Zhang, Y.-Q., see Hu, Z.-D.
B29 (2015) 1550184

B29 (2015) 1450247

B29 (2015) 1540023

B29 (2015) 1450243

B29 (2015) 1542050

B29 (2015) 1542023

B29 (2015) 1550178

B29 (2015) 1540025

B29 (2015) 1550013

B29 (2015) 1540026 B29 (2015) 1540028 B29(2015) 1540003 B29 (2015) 1540027 B29 (2015) 1550095 B29 (2015) 1542008 B29 (2015) 1550163 B29 (2015) 1550100 B29 (2015) 1550227 B29 (2015) 1550185 B29 (2015) 1550064 B29 (2015) 1542010

B29(2015) 1550029 B29 (2015) 1550096 B29 (2015) 1550184 B29 (2015) 1550238

B29 (2015) 1550050

B29(2015) 1550136 B29 (2015) 1550138 B29 (2015) 1542047 B29 (2015) 1542045 B29 (2015) 1550079 B29 (2015) 1542040 B29(2015) 1550138 
Author Index

Zhang, Z.-H., see He, J.-J.

Zhang, Z.-H., see Hu, X.-F.

Zhang, Z.-H., see Kuang, M.-Q.

Zhang, Z.-H., see Xu, Y.-Q.

Zhao, H., see Feng, $\mathrm{S}$.

Zhao, H., see He, H.-L.

Zhao, H., see Kuang, L.

Zhao, L. F., see Jiang, J.

Zhao, X., see He, T.

Zhao, X., see Wu, Y.

Zhao, Y., see Cao, G.

Zhao, Y., see Jiang, J.

Zhao, Y., see Jiang, J.

Zhao, Y., see Zhang, H.

Zheng, B. T., see Che, T.

Zheng, J., see Che, $\mathrm{T}$.

Zheng, J., see Liu, W.

Zheng, J., see Sun, R. X.

Zheng, Q., see Chi, Z.

Zheng, Q., see Guo, X.

Zheng, S., see Wang, S.

Zheng, X.-Y., Zhang, X.-R., Zhang, L.-L. \& Hu, G.-K., Structure stability and magnetic properties of $W_{n} H_{2}(n=7-12)$ clusters

Zheng, Y., see Bian, B.

Zheng, Z. G., see Gao, T. F.

Zhihua, P., Baoxing, L., Yanfeng, P., Peng, J., Xiaogui, Z. \& Ningyu, X., Microwave absorption by hydrogen plasma in carbon nanotubes films with layered dielectric structure

Zhong, K., Xu, G., Zhang, J., Liao, R. \& Huang, Z., Work function change of $\mathrm{Ni}, \mathrm{HfO}_{2}$ films and $\mathrm{Ni} / \mathrm{HfO}_{2}$ interfaces as a function of external electric field

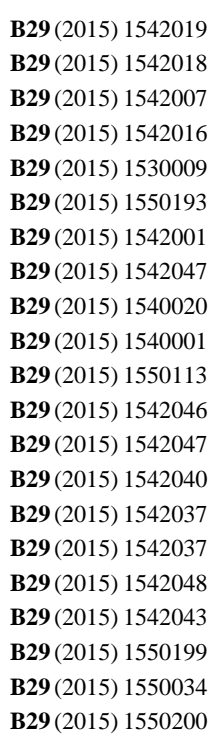

B29 (2015) 1550184 B29 (2015) 1550171 B29 (2015) 1550069

B29(2015) 1550105

B29 (2015) 1550168
Zhou, D. J., see Jiang, J.

Zhou, F. Q., see Wan, M. L.

Zhou, H., see Leng, M.

Zhou, H.-F., see Dong, H.-N.

Zhou, H.-F., see Dong, H.-N.

Zhou, H.-F., see Wang, J.

Zhou, J., Xu, W., Guo, X. \&

Ma, X., Railway faults spreading model based on dynamics of complex network

Zhou, J.-N., see Wang, R.

Zhou, P. B., see Liu, H.

Zhou, W.-X., see Meng, H.

Zhou, X. J., see Bok, J. M.

Zhou, X., see Chen, Q.

Zhou, X.-L., see Wu, X.-L.

Zhou, Z.-W., Sun, J.-X. \& Khan, M. A., Shifted Fang-Howard wavefunction for the twodimensional electron gas and application to MOSFETS

Zhu, H. P., see Zhang, G. P.

Zhu, H., see Liu, L.

Zhu, J., see Xiong, J.

Zhu, J.-M., Quantum phase transitions in composite matrix product states of one-dimensional spin-1/2 chains

Zhu, M. Y., see Li, W. X.

Zhu, Q. S., see Song, Y. F.

Zhu, X. F., see Chen, L. A.

Zhu, Z., Li, Y., Zhang, M. \& Hui, C., Effects of stress concentration on the fatigue strength of 7003-T5 aluminum alloy butt joints with weld reinforcement Zhu, Z., see Bian, B.

Žigas, L., see Audzijonis, A.

Zou, Y., see Liu, L.
B29 (2015) 1542047

B29 (2015) 1550114

B29 (2015) 1550078

B29 (2015) 1542011

B29 (2015) 1542022

B29 (2015) 1542021

B29 (2015) 1550038

B29 (2015) 1550026

B29 (2015) 1542049

B29 (2015) 1550181

B29 (2015) 1542005

B29 (2015) 1542050

B29 (2015) 1550103

B29 (2015) 1450250

B29 (2015) 1550115

B29 (2015) 1550116

B29 (2015) 1550191

B29 (2015) 1550071

B29 (2015) 1542032

B29 (2015) 1550212

B29 (2015) 1550130

B29 (2015) 1540023 B29 (2015) 1550171 B29 (2015) 1550167 B29 (2015) 1550116 